\title{
Breastfeeding and the risk of childhood cancer: a systematic review and dose- response meta-analysis
}

Qing $\mathrm{Su}^{1+}$, Xiaohui Sun ${ }^{1+}$, Liwen Khu $^{2+}$, Qin Yan ${ }^{1}$, Peiwen Zheng ${ }^{3}$, Yingying Mao ${ }^{1 *}$ and Ding Ye ${ }^{1 *}$ (D)

\begin{abstract}
Background: The aim of this study was to quantitatively summarize the available evidence on the association of breastfeeding with the risk of childhood cancer.

Methods: A literature search of PubMed and Embase databases was performed to identify eligible observational studies published from inception to July 17, 2020. The categorical and dose-response meta-analysis was conducted by pooling relative risk (RR) or odds ratio (OR) estimates with $95 \%$ confidence intervals (Cls). Potential sources of heterogeneity were detected by meta-regression and stratification analysis. Sensitivity analysis and publication bias test were also carried out.
\end{abstract}

Results: Forty-five articles involving 475,579 individuals were included in the meta-analysis. Among the thirty-three studies on the association between breastfeeding and risk of childhood leukemia, the pooled risk estimates were 0.77 (95\% Cl, 0.65-0.91) and 0.77 (95\% Cl 0.63-0.94) for ever versus non/occasional breastfeeding and longest versus shortest breastfeeding duration group, respectively. There was clear indication for non-linear dose-response relationship between breastfeeding duration and the risk of childhood leukemia $(P$ non-linear $<0.001)$. The most protective effect $(\mathrm{OR}, 0.66,95 \% \mathrm{Cl} 0.62-0.70)$ was observed at a breastfeeding duration of 9.6 months. Four studies examined, the association between breastfeeding and risk of childhood neuroblastoma, and significant inverse associations were consistently observed in both the comparisons of ever breastfeeding versus non/occasional breastfeeding $(\mathrm{OR}=0.59,95 \% \mathrm{Cl} 0.44-0.81)$ and longest versus shortest breastfeeding $(\mathrm{OR}=0.61,95 \% \mathrm{Cl} 0.44-0.83)$. However, no associations of breastfeeding with risk of other cancers were found.

Conclusions: Our study supports a protective role of breastfeeding on the risk of childhood leukemia, also suggesting a non-linear dose-response relationship. Further studies are warranted to confirm the association between breastfeeding and risk of childhood neuroblastoma.

Keywords: Breastfeeding, Childhood cancer, Meta-analysis, Dose-response

\footnotetext{
*Correspondence: myy@zcmu.edu.cn; yeding@zcmu.edu.cn

${ }^{\dagger}$ Qing Su, Xiaohui Sun and Liwen Zhu contributed equally to this work.

'Department of Epidemiology and Biostatistics, School of Public Health,

Zhejiang Chinese Medical University, 548 Binwen Road, Hangzhou 310053,

China

Full list of author information is available at the end of the article
}

(c) The Author(s). 2021 Open Access This article is licensed under a Creative Commons Attribution 4.0 International License, which permits use, sharing, adaptation, distribution and reproduction in any medium or format, as long as you give appropriate credit to the original author(s) and the source, provide a link to the Creative Commons licence, and indicate if changes were made. The images or other third party material in this article are included in the article's Creative Commons licence, unless indicated otherwise in a credit line to the material. If material is not included in the article's Creative Commons licence and your intended use is not permitted by statutory regulation or exceeds the permitted use, you will need to obtain permission directly from the copyright holder. To view a copy of this licence, visit http://creativecommons.org/licenses/by/4.0/ The Creative Commons Public Domain Dedication waiver (http://creativecommons.org/publicdomain/zero/1.0/) applies to the data made available in this article, unless otherwise stated in a credit line to the data. 


\section{Background}

Childhood cancer is emerging as a major cause of death in children worldwide, which is bleaker for children with cancer in lower-middle-income countries [1]. Although childhood cancer only accounted for $1 \%$ of the total cancer [2], while once it occurs, a range of medical, psychological, ethical, and societal concerns are raised. Moreover, the global age-standardized incidence rates of registered cancers in children aged $0-14$ years have increased from 124.0 to 140.6 per million person-years since the 1980s [3]. To date, little is known about the etiology of childhood cancer, but maternal reproductive health are potential explanations for a fraction of the incidence [4-6].

It is widely accepted that breastfeeding may protect mothers against breast cancer [7] and ovarian cancer [8], but also bring multiple health benefits for the infants [9, 10]. However, the relationships of breastfeeding with the risk of childhood cancer are inconsistent across studies and the associations may differ by cancer types. Several studies have shown that breastfeeding had a protective effect on childhood cancer and the protection increased with the duration of the breastfeeding $[11,12]$. However, some previous studies showed no evidence of protection from breastfeeding for childhood cancer, and the analyses by duration of breastfeeding also failed to support the protective hypothesis $[13,14]$. When specific types of childhood cancer were examined, Amitay and Keinan-Boker [15] showed that breastfeeding was inversely associated with the risk of childhood leukemia. However, Wang et al. [16] provided limited evidence for a protective role of breastfeeding in childhood Hodgkin's lymphoma. Other publications have even reported that prolonged breastfeeding was positively associated with the risk of childhood malignant germ cell tumors [17] and leukemia [18].

A previous meta-analysis of the association between breastfeeding and the risk of childhood cancer was based on 26 original studies published up to June 2004 [19]. The pooled effect estimates suggested that breastfeeding was associated with 9\% (95\% confidence interval (CI), 2$16 \%)$ lower risk of acute lymphoblastic leukemia (ALL), 24\% (95\% CI 3-40\%) lower risk of Hodgkin's disease, and $41 \%$ (95\% CI 22-56\%) lower risk of neuroblastoma, but no associations of breastfeeding with acute nonlymphoblastic leukemia, non-Hodgkin's lymphoma, central nervous system cancers, malignant germ cell tumors, juvenile bone tumors, or other solid cancers. Since then, evidences regarding this association have accumulated rapidly and provided more answers to this question. For the dose-response relationship, a previous pooled analysis showed that the protective effect of breastfeeding on the risk of childhood ALL was lowest at the breastfeeding duration of 8-10 months [20]. However, the exact dose-response relationship has not yet been evaluated for other cancer types. Therefore, we conducted this updated systematic review and dose-response metaanalysis of epidemiological studies to quantify precisely the impact of breastfeeding on the incidence of childhood cancer.

\section{Methods}

The study was registered in the international prospective register of systematic reviews (PROSPERO: CRD42020199446). The Preferred Reporting Items for Systematic reviews and Meta-Analysis (PRISMA) checklist for reporting the metaanalysis was shown in Additional file 1: Table S1.

\section{Search strategy}

Original articles from PubMed and EMBASE databases were systematically searched from the inception to 17 July 2020 to identify potentially eligible studies on the association between breastfeeding and the risk of childhood cancer. The search strategy was as follows: ("child" OR "pediatric" OR "childhood" OR "children") AND ("cancer" OR "tumor" OR "neoplasm" OR "carcinoma" OR "malignancy" OR "leukemia" OR "lymphoma" OR "neuroblastoma" OR "retinoblastoma" OR "melanoma") AND ("breastfeeding" OR "infant feeding" OR "infant nutrition"). In addition, we conducted manual retrieval of the relevant references.

\section{Inclusion and exclusion criteria}

The eligible studies were included as follows: (1) study design of cohort or case-control, (2) clearly defining the outcome of interest as cancer of specific anatomical site [21-23], (3) reporting the relative risk (RR) or odds ratio (OR) and corresponding 95\% CI to calculate the association between breastfeeding and cancer risk among children, or providing sufficient data to calculate them, (4) if study populations overlapped, we selected the one with larger sample size. The exclusion criteria were as follows: (1) systematic review or meta-analysis; (2) letter, meeting, or comment; (3) duplicate studies retrieved from various databases. Two reviewers (QS and XS) independently performed study review and inclusion, and discrepancies were solved by a third reviewer (DY).

\section{Data extraction and quality assessment}

We extracted crucial information from the final studies retained, including first author, year of publication, data collection years, country or region, sample size, age in years, source of participants, cancer site, method of assessing breastfeeding, breastfeeding category, variables adjusted or matched, and corresponding risk estimates with $95 \%$ CIs.

Two researchers (QY and PZ) independently rated the quality of the included studies using the Newcastle- 
Ottawa Scale with scores ranging from 0 to 9 points [24]. This scale evaluates studies on the following aspects: (I) selection of cases and controls (4 scores); (II) comparability of cases and controls (2 scores); (III) ascertainment of exposure and non-response rate (3 scores). Studies with a quality score more than 7 points were considered as high quality. Two researchers (QS and QY) independently assessed the potential risk of bias using the risk of bias in nonrandomized studies of interventions (ROBINS-I) tool [25]. This tool encompasses seven domains: the presence of any confounding variables, selection bias, deviations from the exposure, misclassification of the exposure, missing data, measurement of outcomes, and selection of the reported results. In this approach, a study was categorized as "low risk," "moderate risk," "serious risk," or "critical risk" of bias.

\section{Statistical analysis}

All statistical analyses were performed using STATA version 14.0. The multivariate-adjusted risk estimates were selected if they were reported in the original article; otherwise, the unadjusted risk estimates were calculated using the original data. For the studies reported risk estimates relative to a reference category other than shortest breastfeeding duration, the risk estimates were recalculated using the shortest breastfeeding duration as reference by using the Orsini method [26]. When pooling the risk estimates, we regraded the shortest breastfeeding duration as the reference group (non/occasional breastfeeding), i.e., some defined never and some defined less than 1 month, 2 months, or 6 months.

In the analysis of breastfeeding versus non/occasional breastfeeding, if the corresponding estimate had not been presented in a study, estimates associated with different breastfeeding categories were synthesized into a single estimate. We also combined the risk estimates comparing the longest with the shortest breastfeeding duration among the studies with equal or greater than three different breastfeeding categories. The forest plot of the association between breastfeeding and the risk of childhood cancer was generated for breastfeeding and non/occasional breastfeeding and longest versus shortest breastfeeding duration, respectively.

A two-stage dose-response meta-analysis [27] was conducted to investigate the potential non-linear doseresponse relationship between breastfeeding and the risk of childhood cancer. Briefly, a restricted cubic splines model with four knots at fixed percentiles, 5\%, 35\%, $65 \%$, and $95 \%$, of exposure level was used, which had negligible influence on the estimates. We assigned a null value to the lower bound of the reference group. The midpoint of the range was adopted to represent the category. When the category was open-ended, we assigned the midpoint of the upper open-ended category assuming that they had the same interval as the adjacent category.

The heterogeneity was evaluated by Q-statistic test and $I$-squared $\left(I^{2}\right)[28,29]$. The random-effects model was used to pool the effect estimates, as the approach can be used whether or not there is heterogeneity [30]. Subgroup analyses were performed by year of publication, geographic location, quality score, sample size, study design, and definition of reference category. Heterogeneity between strata by the above stratified factors was assessed by meta-regression analysis. Sensitivity analysis was performed to evaluate the effect of a particular study on the overall results by deleting one study at a time and combining the effect values of the remaining studies.

Funnel plot was generated, and the symmetry means no potential publication bias. Publication bias was assessed using Egger's test [31] and Begg's test [32].

\section{Results}

\section{Systematic search}

The flowchart of study selection is presented in Additional file 1: Figure S1. The primary search strategy for PubMed and Embase yielded 2905 and 2771 articles, and manual search from the reference lists of original studies or relevant reviews and meta-analyses on this topic yielded 67 additional articles. After removal of duplicates, 5545 articles were retrieved for assessment based on title and abstract, of which 116 articles were included for full text evaluation. After exclusion of 8 articles with insufficient data, 4 articles only reporting data on all cancers, 47 articles as meta, review, comment or meeting abstract, 2 articles conducted not among children, and 10 articles with overlapping data sets (Additional file 1: Table S2), a total of 45 articles with 475,579 participants were included for this meta-analysis [13, 14, 17, 18, 33-73].

\section{Characteristics of the included studies}

The characteristics of selected studies are shown in Table 1. The total number of participants (from 140 to $410,147)$ varied widely across the included studies. All articles represented a range of geographical areas in Europe $(n=20)$, Asia $(n=8)$, and North America or Oceania $(n=17)$. There were 32 articles with populationbased case-control design, 12 articles with hospital-based case-control design, and one article with cohort design. The median quality score of all included articles was 7 , which resulted in 33 articles with a score of 7 or more and 12 articles with a score less than 7. Based on the ROBINS-I tool, 37 studies were considered at moderate risk of bias, and 8 studies were rated at serious risk of bias. Of the 45 articles, 33 studies provided the effect estimates for the association of breastfeeding on leukemia, 11 studies on lymphoma, 7 studies on brain tumors, 4 


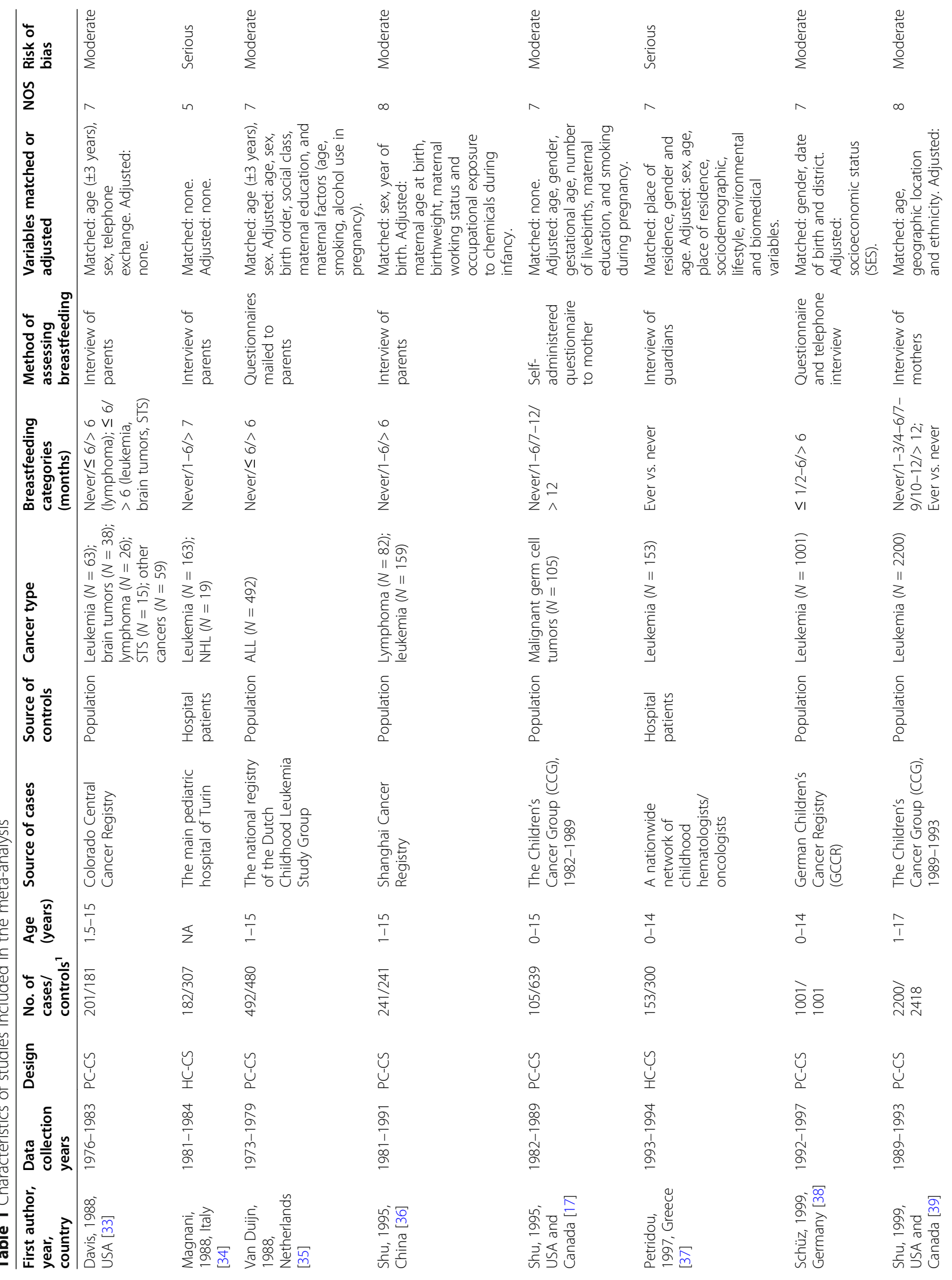









\begin{tabular}{|c|c|c|c|c|c|c|c|}
\hline 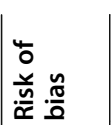 & $\begin{array}{l}\frac{0}{0} \\
\frac{0}{0} \\
\frac{0}{0} \\
\frac{0}{2}\end{array}$ & $\begin{array}{l}\stackrel{n}{o} \\
\stackrel{0}{\bar{n}} \\
\sim \sim n\end{array}$ & $\begin{array}{l}\frac{0}{\pi} \\
\frac{\pi}{\pi} \\
\frac{0}{2} \\
\sum\end{array}$ & $\begin{array}{l}\frac{0}{\pi} \\
\frac{\pi}{\pi} \\
\frac{0}{0} \\
\sum\end{array}$ &  & $\begin{array}{l}\frac{0}{\pi} \\
\frac{\pi}{\pi} \\
\frac{0}{2} \\
\sum\end{array}$ & $\begin{array}{l}\frac{0}{\pi} \\
\frac{\pi}{\pi} \\
\frac{0}{0} \\
\frac{0}{2}\end{array}$ \\
\hline 气ั & 0 & 0 & $\infty$ & $\wedge$ & $\wedge$ & $\wedge$ & $\wedge$ \\
\hline 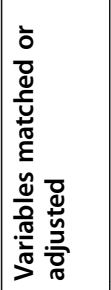 & 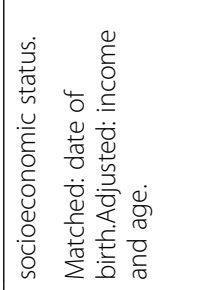 &  & 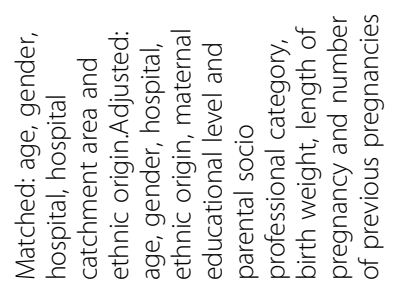 & 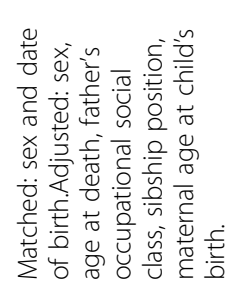 & 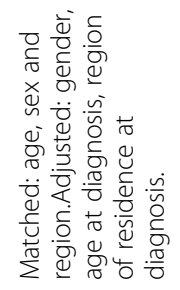 & 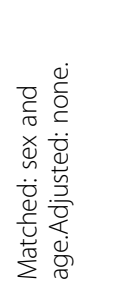 & 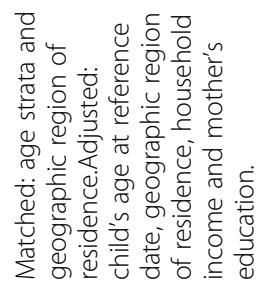 \\
\hline 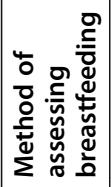 & 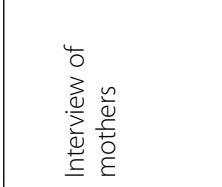 & 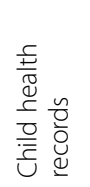 & 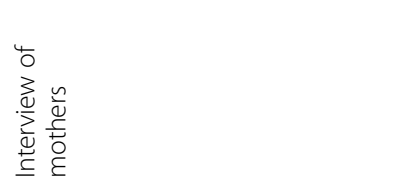 & 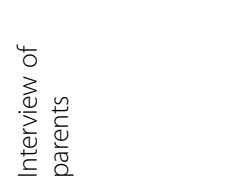 & 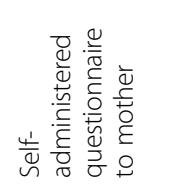 & 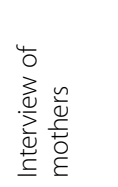 & 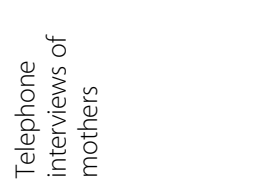 \\
\hline 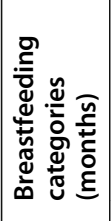 & 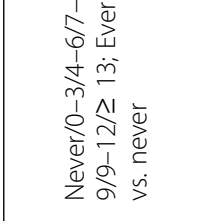 & 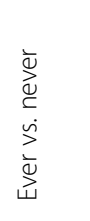 & 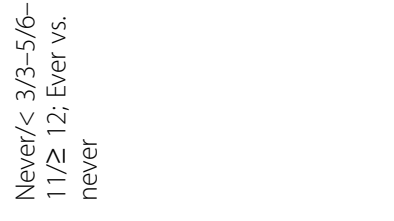 & 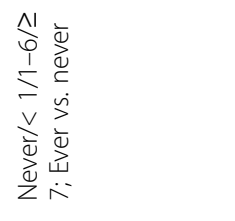 & 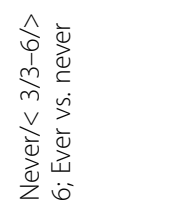 & $\begin{array}{l}0 \\
\hat{1} \\
\dot{3} \\
0 \\
0\end{array}$ & 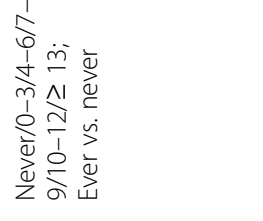 \\
\hline 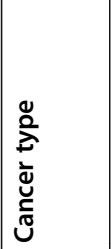 & 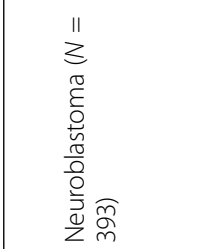 & 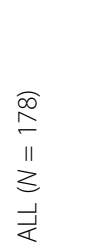 & 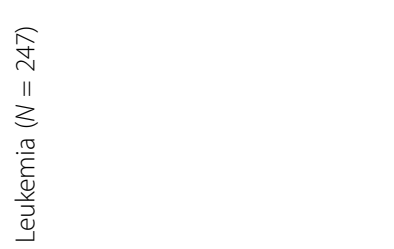 & 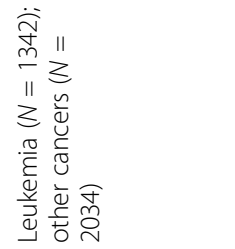 & 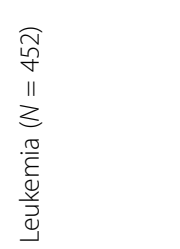 & 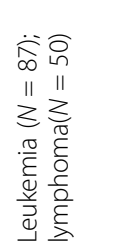 & 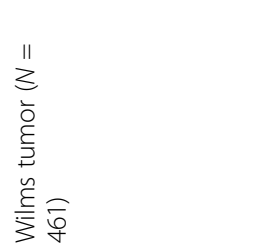 \\
\hline 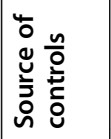 & $\begin{array}{l}\frac{1}{0} \\
\frac{0}{0} \\
\frac{\pi}{2} \\
\frac{0}{0} \\
0\end{array}$ & $\begin{array}{l}\frac{c}{0} \\
\frac{0}{\bar{t}} \\
\frac{\pi}{2} \\
\frac{0}{0} \\
0\end{array}$ &  & $\begin{array}{l}\frac{c}{0} \\
\frac{0}{0} \\
\frac{0}{2} \\
\frac{0}{0} \\
0\end{array}$ & $\begin{array}{l}\frac{.}{0} \\
\frac{0}{0} \\
\frac{\overline{0}}{\overline{0}} \\
\frac{0}{0}\end{array}$ & $\begin{array}{l}\frac{c}{2} \\
\frac{0}{0} \\
\frac{0}{2} \\
\frac{0}{0} \\
0\end{array}$ & $\begin{array}{l}\frac{c}{.0} \\
\frac{0}{0} \\
\frac{0}{2} \\
\frac{0}{0} \\
0\end{array}$ \\
\hline 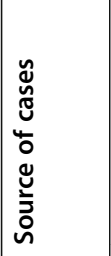 & 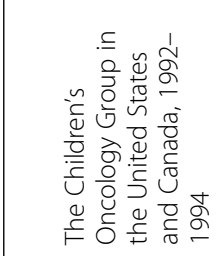 & 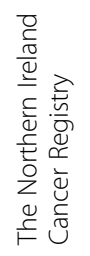 &  & 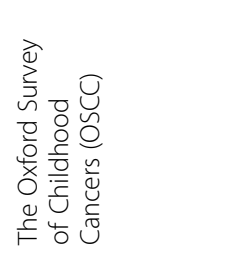 &  &  & 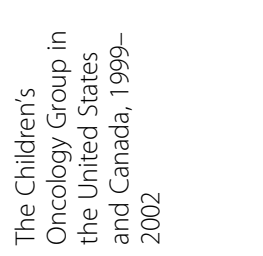 \\
\hline g & 定 & $\frac{0}{0}$ & 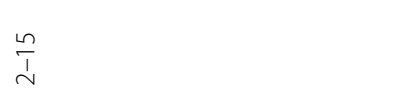 & $\stackrel{ \pm}{I}$ & $\stackrel{\mathscr{L}}{I}$ & $\frac{0}{I}$ & 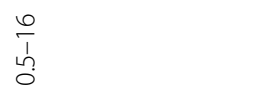 \\
\hline 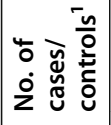 & 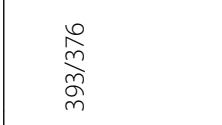 & 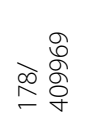 &  & $\begin{array}{l}\text { dे } \\
\text { లె }\end{array}$ & 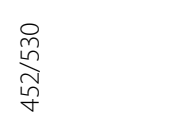 & 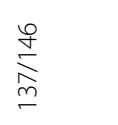 & $\frac{\frac{m}{j}}{\frac{f}{q}}$ \\
\hline 产 & 苍 & 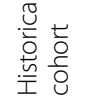 & $\begin{array}{l}\breve{u}^{u} \\
\text { I }\end{array}$ & 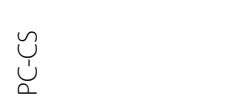 & 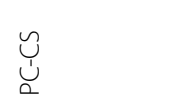 & 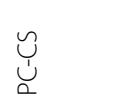 & 范 \\
\hline 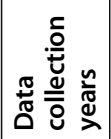 & 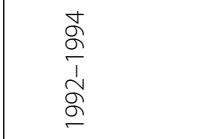 & 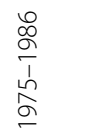 & $\begin{array}{l}\sigma \\
\alpha \\
\sigma \\
\vdots \\
\alpha \\
\alpha\end{array}$ & $\begin{array}{l}\bar{\infty} \\
o \\
\stackrel{1}{1} \\
\stackrel{1}{\alpha}\end{array}$ & 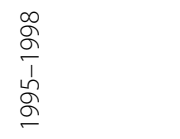 & $\begin{array}{l}8 \\
8 \\
1 \\
\vdots \\
\text { o } \\
-\end{array}$ & 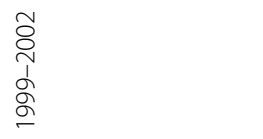 \\
\hline 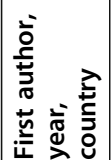 & 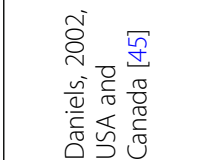 & 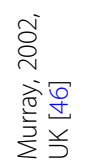 & 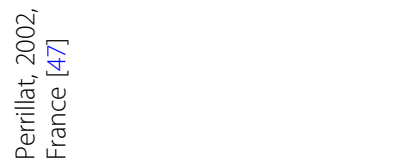 & 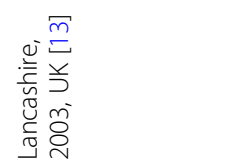 & 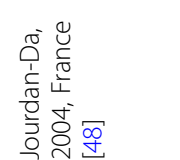 & 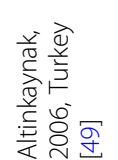 & 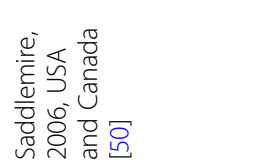 \\
\hline
\end{tabular}









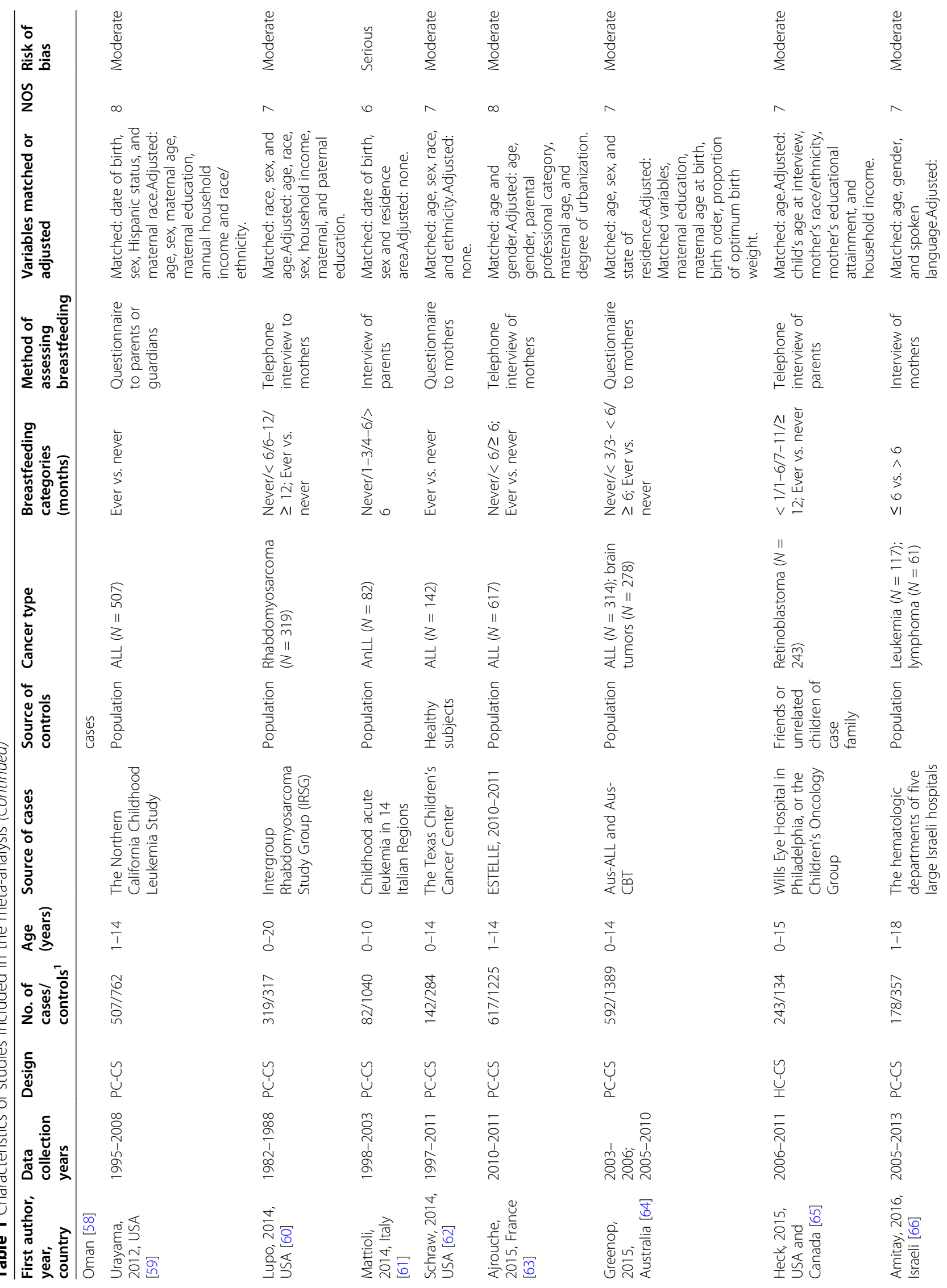




\begin{tabular}{|c|c|c|c|c|c|c|c|c|}
\hline 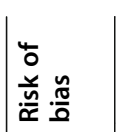 & $\begin{array}{l}\frac{0}{\pi} \\
\frac{\pi}{0} \\
\frac{0}{0} \\
\sum\end{array}$ & $\begin{array}{l}\frac{\tilde{y}}{0} \\
\frac{\pi}{\overline{0}} \\
\frac{0}{0} \\
\sum\end{array}$ & $\begin{array}{l}\frac{0}{0} \\
\frac{0}{0} \\
\frac{0}{0} \\
\sum\end{array}$ & $\begin{array}{l}\frac{0}{\pi} \\
\frac{\pi}{0} \\
\frac{\pi}{0} \\
\sum\end{array}$ & 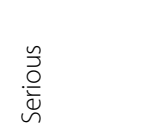 & 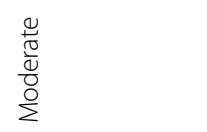 & $\begin{array}{l}\stackrel{n}{a} \\
\stackrel{0}{\bar{N}}\end{array}$ & $\begin{array}{l}\frac{0}{\pi} \\
\frac{\pi}{0} \\
\frac{\pi}{0} \\
\sum\end{array}$ \\
\hline 气̆ & $\infty$ & $\infty$ & $\wedge$ & $\infty$ & in & in & & $\wedge$ \\
\hline 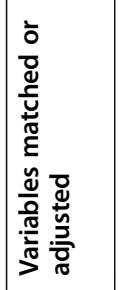 & 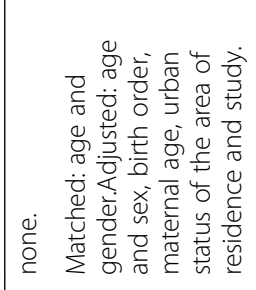 & 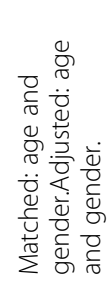 & 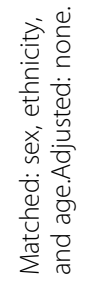 & 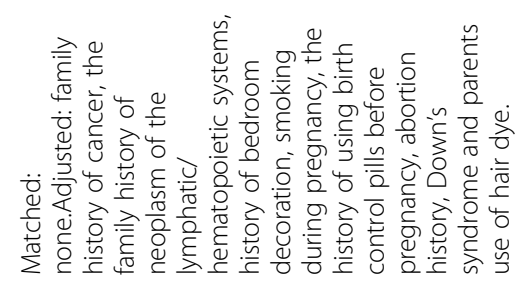 & 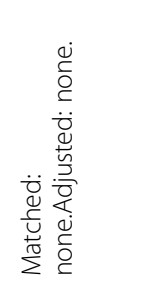 & 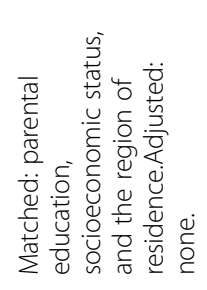 & 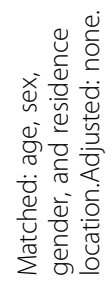 & 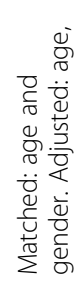 \\
\hline 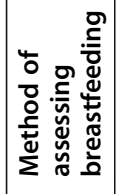 & 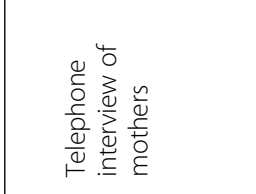 &  & 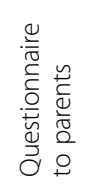 & 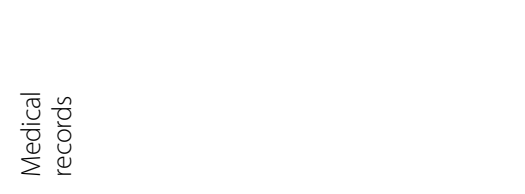 & 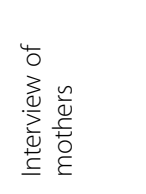 & 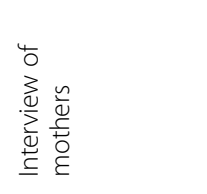 & 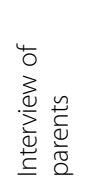 & 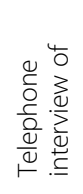 \\
\hline 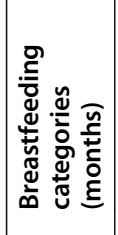 & 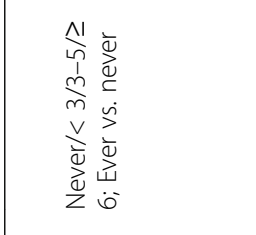 & 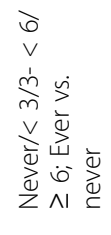 & 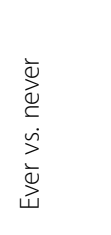 & 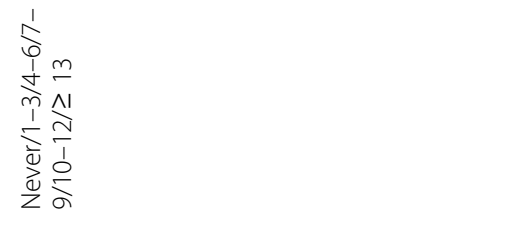 &  & 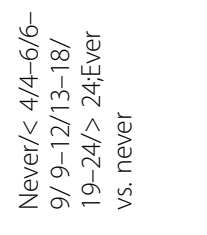 & 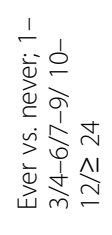 & 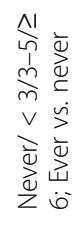 \\
\hline 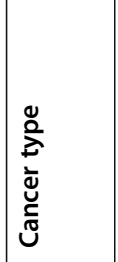 & 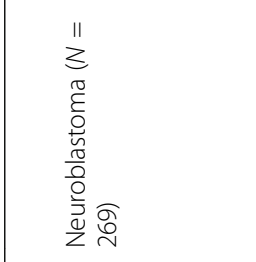 & 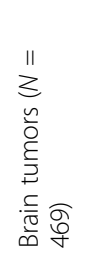 & $\begin{array}{l}\bar{\equiv} \\
\frac{1}{11} \\
\vdots \\
\vec{z} \\
\vec{z}\end{array}$ & 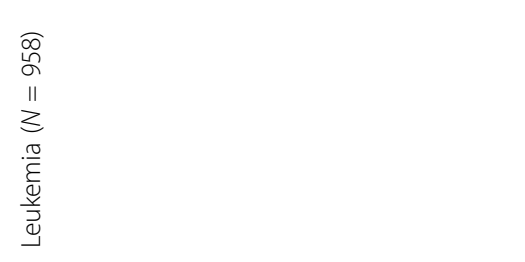 & 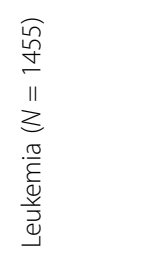 &  & $\begin{array}{l}\stackrel{\sqrt{2}}{\frac{11}{11}} \\
3 \\
\vec{⿱}\end{array}$ & 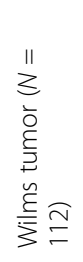 \\
\hline  & $\begin{array}{l}\frac{c}{\circ} \\
\frac{0}{0} \\
\frac{0}{2} \\
\frac{0}{0} \\
0\end{array}$ & $\begin{array}{l}\frac{c}{\circ} \\
\frac{0}{0} \\
\frac{0}{\overline{0}} \\
\frac{0}{0} \\
0\end{array}$ & $\begin{array}{l}\frac{.}{\circ} \\
\frac{0}{0} \\
\frac{0}{\overline{0}} \\
\frac{0}{0} \\
0\end{array}$ &  & 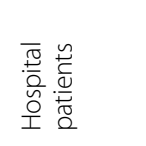 & 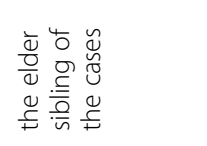 & 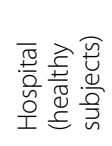 & $\begin{array}{l}\frac{c}{\circ} \\
\frac{0}{0} \\
\frac{0}{3} \\
0 \\
0\end{array}$ \\
\hline 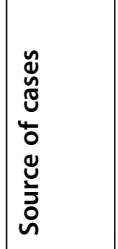 &  & 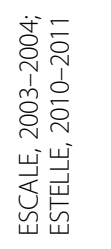 & 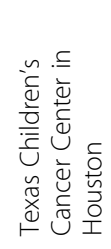 &  & 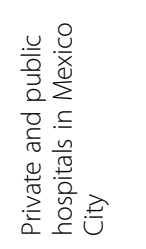 & 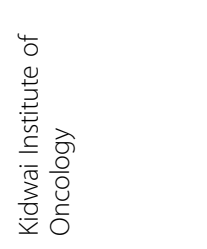 & 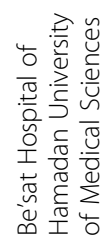 & 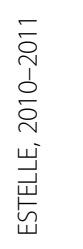 \\
\hline 离 & \begin{tabular}{l}
0 \\
\multirow{1}{0}{} \\
0
\end{tabular} & $\frac{I}{I}$ & $\frac{d}{\partial}$ & $\frac{t}{d}$ & 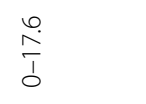 & $\stackrel{\stackrel{n}{I}}{I}$ & $\stackrel{I}{I}$ & $\begin{array}{l}\bar{\tau} \\
\stackrel{1}{n} \\
0\end{array}$ \\
\hline 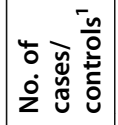 & 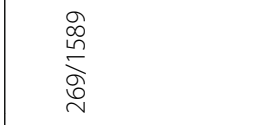 & $\begin{array}{l}\stackrel{a}{\Lambda} \\
\substack{\delta \\
y}\end{array}$ & $\stackrel{\substack{\mathfrak{m} \\
\Sigma}}{\Sigma}$ & $\begin{array}{l}\stackrel{\sim}{\infty} \\
\stackrel{\infty}{\infty} \\
\infty \\
\alpha \\
\infty\end{array}$ & 冠虽 & 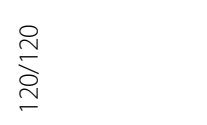 & $\stackrel{\stackrel{一}{\leftrightarrows}}{\stackrel{m}{\leftrightarrows}}$ & 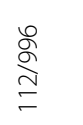 \\
\hline 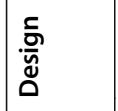 & 菭 & $\breve{u}_{0}$ & 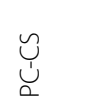 & $\begin{array}{l}\breve{U} \\
\stackrel{U}{I}\end{array}$ & $\begin{array}{l}\breve{U} \\
\stackrel{U}{I}\end{array}$ & $\begin{array}{l}y_{\mathcal{U}} \\
\text { I }\end{array}$ & $\begin{array}{l}\breve{u} \\
\stackrel{u}{I}\end{array}$ & $\begin{array}{l}y_{u} \\
\alpha\end{array}$ \\
\hline  & 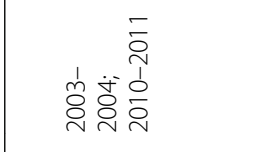 &  & 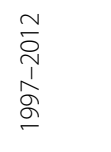 & $\begin{array}{l}\hat{a} \\
\overline{1} \\
o \\
o \\
o \\
1\end{array}$ &  & $\stackrel{n}{\sigma}$ & 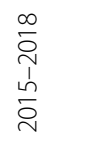 & $\begin{array}{l}\infty \\
\stackrel{\infty}{2} \\
i \\
\stackrel{\omega}{2} \\
i\end{array}$ \\
\hline 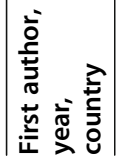 & 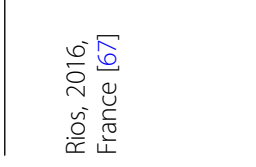 & 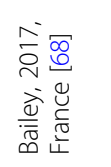 &  & 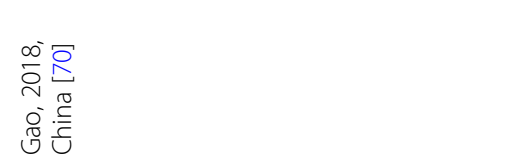 & 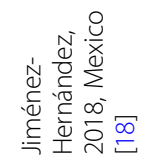 & 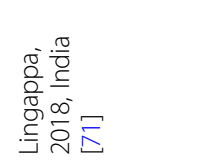 & 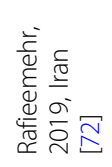 & 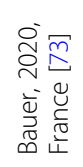 \\
\hline
\end{tabular}




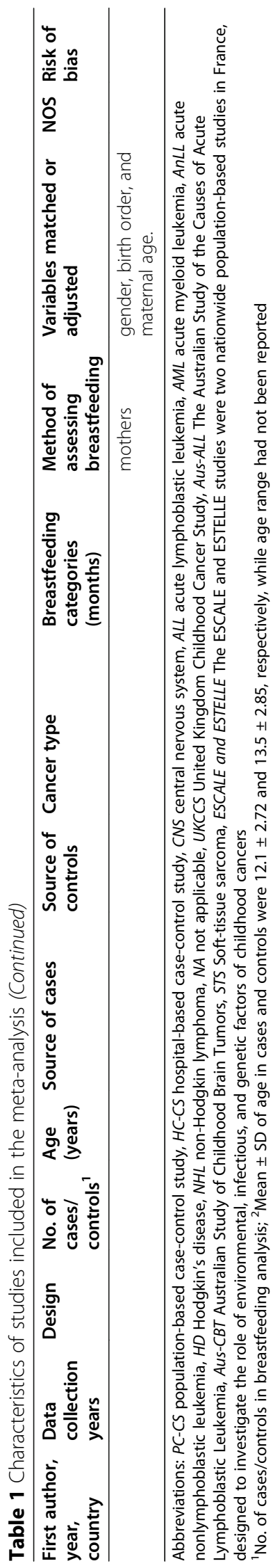


studies on neuroblastoma, 4 studies on soft-tissue sarcoma, 3 studies on nephroblastoma, 2 studies on retinoblastoma, and 2 studies on germ cell tumors. The details of included studies for the subsequent subgroup analysis are shown in Additional file 1: Table S3. Moreover, after excluding studies with the breastfeeding only as dichotomous variable, and no sufficient data of the number of cases and controls in each breastfeeding category, 23 studies were included for leukemia $[13,14,18,34-36$, $38-43,46,47,54,56,58,61,63,64,70-72], 6$ studies for lymphoma [14, 33, 36, 40, 43, 46], and 6 studies for brain tumors $[14,40,44,51,64,67]$ in dose-response meta-analysis.

\section{Leukemia}

Summary of main findings The summary risk estimates of the risk of childhood leukemia were 0.77 (95\% CI, 0.65-0.91) for breastfeeding versus non/occasional breastfeeding and 0.77 (95\% CI, 0.63-0.94) for longest versus shortest breastfeeding duration (Fig. 1). A significant non-linear dose-response relationship between breastfeeding and the risk of childhood leukemia was found $(P<0.001$ for non-linearity; Fig. 2). The overall dose-response relationship approximated to a U-shaped curve. Compared with never breastfeeding, the risk of leukemia was statistically significant at a duration of 4.4-15.0 months. The most protective effect $(\mathrm{OR}=0.66$, 95\% CI 0.62-0.70) was observed at a duration of 9.6 months. Specifically, the average breastfeeding duration of 6 months and 12 months reduced 20\% (95\% CI 15\%$25 \%)$ and $27 \%(95 \%$ CI $22 \%-33 \%)$ of the risk of childhood leukemia, respectively. Begg's funnel plot was presented in Additional file 1: Figure S2. The shape of the funnel plot revealed no asymmetric distribution with a $P$ value of 0.075 by Begg's test and 0.173 by Egger's test.

Subgroup analysis and meta-regression analysis In the subgroup analysis for the comparison of breastfeeding versus non/occasional breastfeeding, there were significant differences in the heterogeneity between strata by geographic location $(P$ in meta-regression $=0.016)$ and definition of reference category $(P$ in metaregression $=0.025)$ (Table 2$)$. Protective effect of breastfeeding on the risk of childhood leukemia cancer was found in population from Asia (OR, 0.43; 95\% CI, 0.190.98), but not Europe and North American or Oceania. The inverse association was more pronounced among the studies with defining occasional breastfeeding as reference group (OR, 0.47; 95\% CI, 0.28-0.80), especially for the studies using breastfeeding less than 6 months as reference group $(\mathrm{OR}=0.49,95 \%$ CI 0.35-0.67). Subgroup analysis of publication year showed similar results with main analysis, and studies with small and larger sample size showed slightly different results. Significant associations were found in studies with high-quality score (OR, 0.72; 95\% CI, 0.59-0.87) and populationbased case-control studies (OR, 0.86; 95\% CI, 0.76-0.96).

We also conducted subgroup analysis stratified by histologic type and found that breastfeeding was associated with a decreased risk for ALL at borderline statistical significance $(\mathrm{OR}=0.92,95 \% \mathrm{CI} 0.84-1.00)$, but not associated with acute myeloid leukemia (AML) risk (Table 2). Moreover, all subgroup analysis of ALL were consistent with those of childhood leukemia (Additional file 1: Table S4). Further stratified by immunophenotype in ALL, the results showed a weak evidence of borderline statistical significance that breastfeeding was associated with a small reduction in B cell ALL risk, but no evidence of the association between breastfeeding and risk of $\mathrm{T}$ cell ALL (Table 2). The forest plots of subgroup analysis are shown in Additional file 1: Figure S3.

Sensitivity analysis The one-study-removed analysis showed that exclusion of each study did not significantly change the results (Additional file 1: Figure S4). Considering that the incidence of childhood leukemia varies with age, which may have an influence on the risk estimate, we performed the repeated analyses by only including the group of studies among children aged 0-14 years old. Consequently, we found that there was no significant effect on the pooled risk estimates (Additional file 1: Figure S5).

\section{Lymphoma}

Summary of main findings There was no suggestive evidence of the association between breastfeeding and risk of childhood lymphoma, with the pooled risk estimates of 0.83 (95\% CI, 0.68-1.02) and 0.77 (95\% CI, 0.53-1.10) for the comparison of breastfeeding versus non/occasional breastfeeding and longest versus shortest breastfeeding duration, respectively (Fig. 3). The nonlinear dose-response relationship curve showed that the association between breastfeeding and the risk of childhood lymphoma was significant in a narrow range of breastfeeding duration $(P=0.046$ for non-linearity; Additional file 1: Figure S6). The funnel plot showed symmetry distribution with a $P$ value of 0.533 by Begg's test and 0.267 by Egger's test (Additional file 1: Figure S7).

Subgroup analysis and meta-regression analysis Heterogeneity between strata by geographic location $(P=$ $0.041)$ and definition of reference group $(P=0.044)$ was identified by meta-regression analysis (Table 3 ). Only the studies conducted in Asia indicated a significant association between breastfeeding and risk of childhood lymphoma $(\mathrm{OR}=0.53,95 \% \mathrm{CI}$ 0.37-0.76). In the 


\section{A. Breasteeding vs. non/occasional breastfeeding}

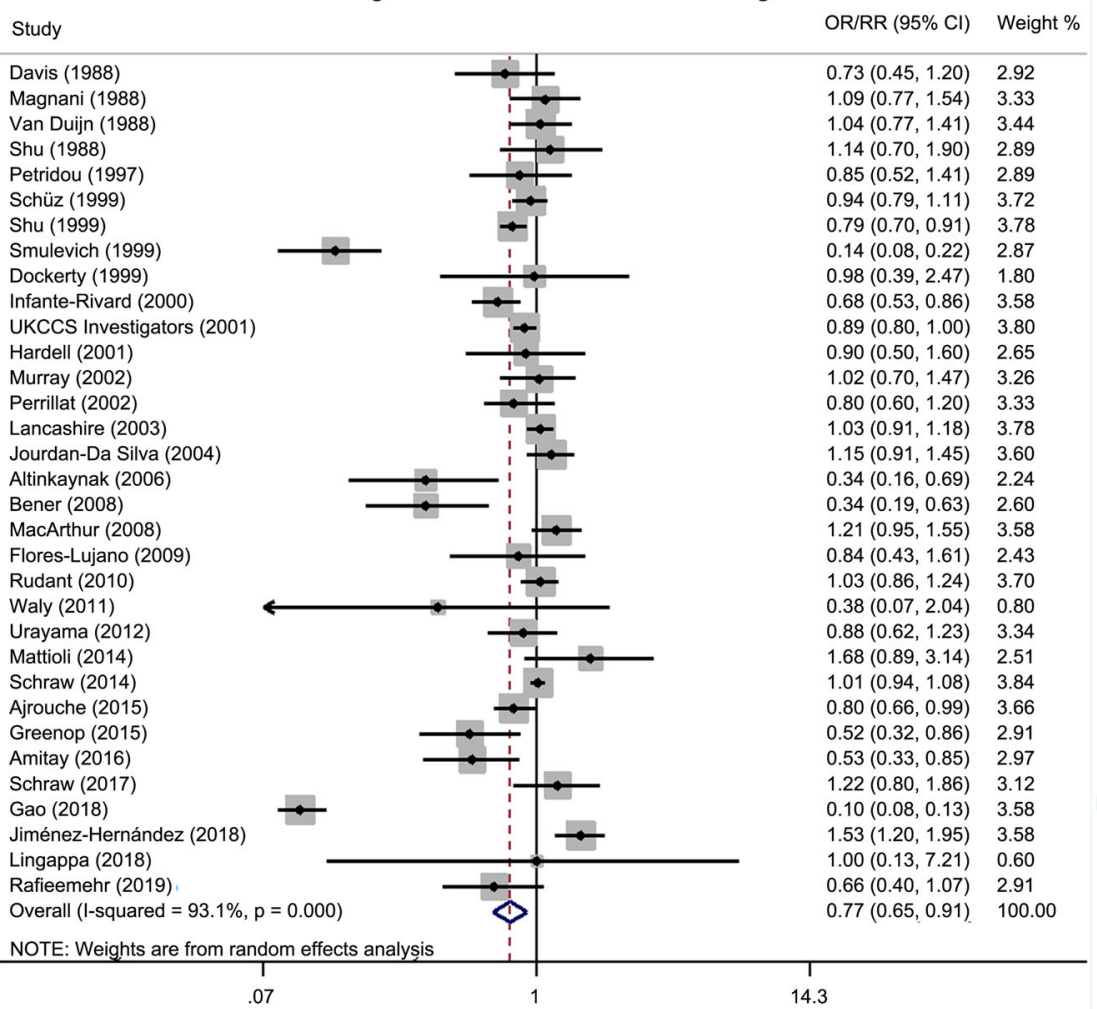

\section{B. Longest vs. shortest bteastfeeding duration}

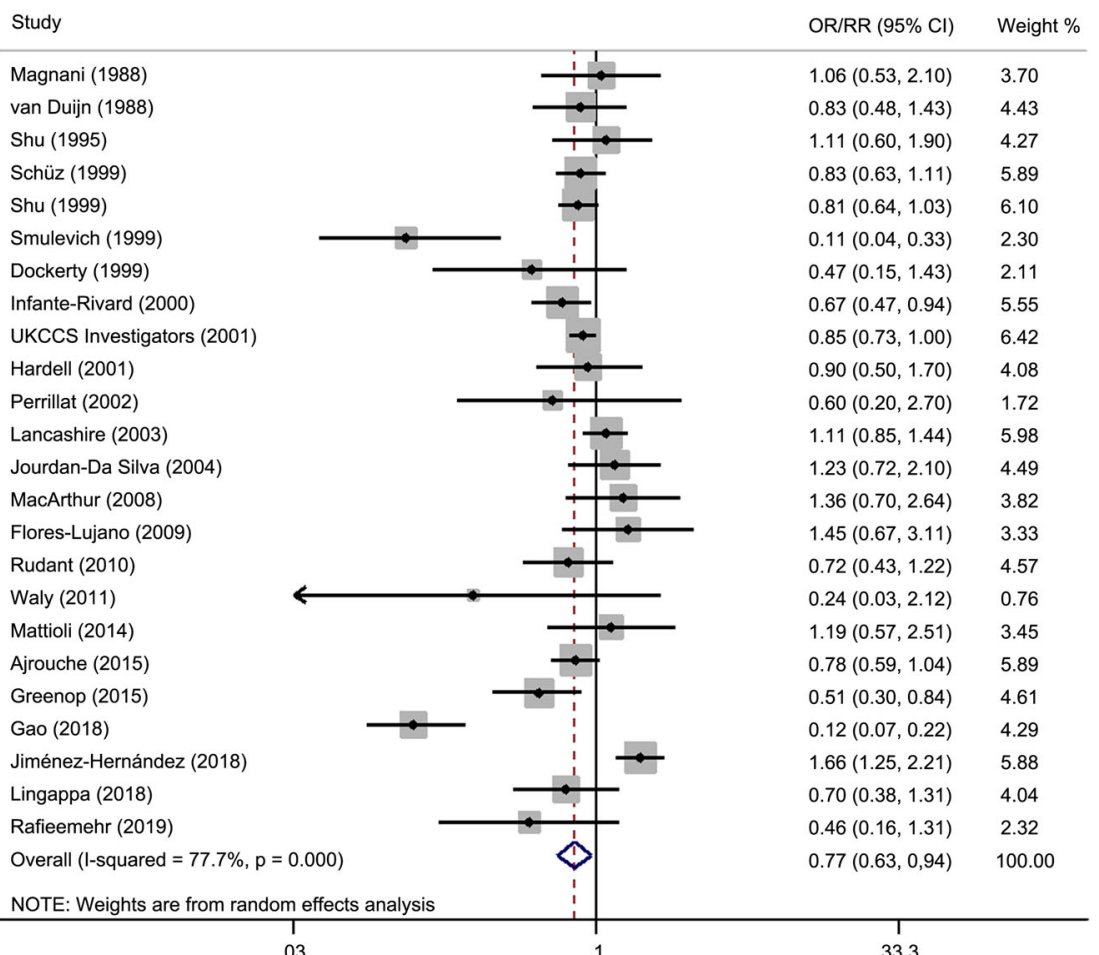

Fig. 1 Forest plots for pooled risk estimates and the corresponding 95\% confidence intervals (Cls) of childhood leukemia risk for a breastfeeding vs. non/occasional breastfeeding and $\mathbf{b}$ longest vs. shortest breastfeeding duration 


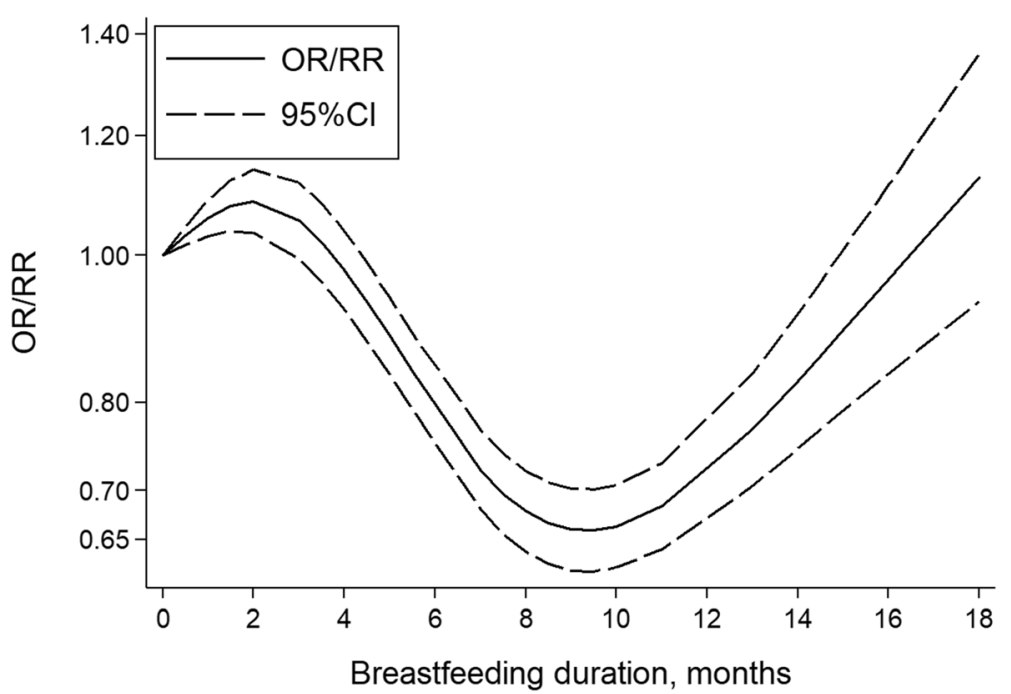

Fig. 2 Risk estimates (solid line) and the corresponding 95\% Cls (dash lines) for the dose-response relationship between breastfeeding and the risk of childhood leukemia

subgroup of studies with defining occasional breastfeeding as reference category, the association between breastfeeding and risk of childhood lymphoma was significant $(\mathrm{OR}=0.66,95 \%$ CI 0.44-0.99). Comparing with the breastfeeding duration of less than 6 months, the pooled risk estimate of prolong breastfeeding for the risk of lymphoma was 0.50 (95\% CI 0.34-0.75). Moreover, there were no significant associations of breastfeeding with both Hodgkin's lymphoma and non-Hodgkin's lymphoma (Table 3). The forest plots of stratified analysis are shown in Additional file 1: Figure S8.

Sensitivity analysis Omitting the studies of UKCCS Investigators et al. [43] and Hardell et al. [14] modified the pooled risk estimates in sensitivity analysis, suggesting the results may be unstable (Additional file 1: Figure S9). Sensitivity analysis by only including the group of studies among children aged 0-14 years old showed the pooled risk estimate of 0.98 (95\% CI 0.84-1.15; Additional file 1: Figure S10).

\section{Brain tumors}

No significant association between breastfeeding and risk of childhood brain tumors was found (Fig. 4). Nonlinear dose-response relationship was also not observed $(P=0.776$ for non-linearity). There was no sign of asymmetry with a $P$ value of 0.764 by Begg's test and 0.261 by Egger's test (Additional file 1: Figure S11). All subgroups showed no significant association between breastfeeding and risk of childhood brain tumors (Table 4). The corresponding forest plots of subgroup analysis are presented in Additional file 1: Figure S12. Sensitivity analysis excluding each study did not substantially change the results (Additional file 1: Figure S13). Sensitivity analysis by excluding the studies considering upper age limits equal and higher than 15 years yielded similar result with the pooled risk estimate of 0.99 (95\% CI 0.87-1.12; Additional file 1: Figure S14).

\section{Neuroblastoma, soft-tissue sarcoma, nephroblastoma, retinoblastoma, and germ cell tumors}

The associations of breastfeeding with risk of childhood neuroblastoma, soft-tissue sarcoma, nephroblastoma, retinoblastoma, and germ cell tumors are presented in Table 5. Significant association was consistently found in neuroblastoma for the two comparisons of breastfeeding versus non/occasional breastfeeding ( $\mathrm{OR}=0.59,95 \% \mathrm{CI}$ $0.44-0.81)$ and longest versus shortest breastfeeding $(\mathrm{OR}=0.61,95 \%$ CI $0.44-0.83)$.

\section{Discussion}

Study quality and study design

Of the 45 studies included, 33 were sufficient to provide at least fair quality evidence regarding the association between breastfeeding and the risk of childhood cancer. The included studies are at risk for selection bias for cases and controls and the potential misclassification introduced by the lack of specificity in exposure definition. Confounding is also an important consideration on account of the nature of observational studies. Almost all studies matched cases with controls by sex (33/45) and age (37/45), and most studies also matched participants using geographic location (15/45), and a few additionally used race or ethnicity $(6 / 45)$. These matching variables are crucial for the comparability of cases and controls. Moreover, parental socioeconomic status (SES) was 
Table 2 Subgroup analysis of association between breastfeeding and childhood leukemia risk for the comparison of breastfeeding versus non/occasional breastfeeding

\begin{tabular}{|c|c|c|c|c|c|c|c|}
\hline & $\begin{array}{l}\text { No. of } \\
\text { studies }\end{array}$ & $\begin{array}{l}\text { No. of } \\
\text { cases }\end{array}$ & OR $(95 \% \mathrm{Cl})$ & $\begin{array}{l}P \\
\text { value }\end{array}$ & $\begin{array}{l}I^{2} \\
(\%)\end{array}$ & $\begin{array}{l}P \text { value for } \\
\text { heterogeneity }\end{array}$ & $\begin{array}{l}P \text { in meta- } \\
\text { regression }\end{array}$ \\
\hline Cancer type & & & & & & & 0.629 \\
\hline AML (A.1) & 11 & 1241 & $\begin{array}{l}0.96(0.79 \sim \\
1.18)\end{array}$ & 0.723 & 38.9 & $<0.001$ & \\
\hline ALL (A.2) & 27 & 11,554 & $\begin{array}{l}0.92(0.84 \sim \\
1.00)\end{array}$ & 0.057 & 66.4 & $<0.001$ & 0.500 \\
\hline B cell ALL (A.3) & 6 & 3974 & $\begin{array}{l}0.91(0.81 \sim \\
1.01)\end{array}$ & 0.070 & 47.3 & 0.350 & \\
\hline T cell ALL (A.4) & 4 & 472 & $\begin{array}{l}1.00(0.79 \sim \\
1.26)\end{array}$ & 0.996 & 8.6 & 0.091 & \\
\hline Year of publication & & & & & & & 0.921 \\
\hline In and before 2000 (A.5) & 10 & 5018 & $\begin{array}{l}0.76(0.59 \sim \\
0.97)\end{array}$ & 0.030 & 84.7 & $<0.001$ & \\
\hline After 2000 (A.6) & 23 & 10,135 & $\begin{array}{l}0.77(0.62 \sim \\
0.97)\end{array}$ & 0.023 & 94.5 & $<0.001$ & \\
\hline Geographic location & & & & & & & 0.016 \\
\hline Europe (A.7) & 14 & 7518 & $\begin{array}{l}0.89(0.77 \sim \\
1.04)\end{array}$ & 0.147 & 81.2 & $<0.001$ & \\
\hline Asia (A.8) & 8 & 1739 & $\begin{array}{l}0.43(0.19 \sim \\
0.98)\end{array}$ & 0.044 & 94.1 & $<0.001$ & \\
\hline $\begin{array}{l}\text { North America or Oceania } \\
\text { (A.9) }\end{array}$ & 11 & 5896 & $\begin{array}{l}0.93(0.79 \sim \\
1.09)\end{array}$ & 0.386 & 77.6 & $<0.001$ & 0.259 \\
\hline North America only (A.10) & 8 & 3285 & $\begin{array}{l}1.01(0.84 \sim \\
1.21)\end{array}$ & 0.960 & 74.0 & $<0.001$ & \\
\hline Oceania only (A.11) & 2 & 411 & $\begin{array}{l}0.63(0.36 \sim \\
1.12)\end{array}$ & 0.113 & 29.0 & 0.235 & \\
\hline Both ${ }^{1}$ (A.12) & 1 & 2200 & $\begin{array}{l}0.79(0.69 \sim \\
0.90)\end{array}$ & $<0.001$ & NA & NA & \\
\hline Study quality score & & & & & & & 0.280 \\
\hline$<7$ (A.13) & 10 & 2752 & $\begin{array}{l}0.97(0.74 \sim \\
1.28)\end{array}$ & 0.827 & 70.5 & $<0.001$ & \\
\hline$\geq 7$ (A.14) & 23 & 12,401 & $\begin{array}{l}0.72(0.59 \sim \\
0.87)\end{array}$ & 0.001 & 94.7 & $<0.001$ & \\
\hline Sample size & & & & & & & 0.787 \\
\hline$\leq 500($ A.15) & 15 & 1938 & $\begin{array}{l}0.77(0.64 \sim \\
0.93)\end{array}$ & 0.007 & 59.3 & 0.002 & \\
\hline$>500($ A.16) & 18 & 13,215 & $\begin{array}{l}0.78(0.61 \sim \\
1.01)\end{array}$ & 0.061 & 95.9 & $<0.001$ & \\
\hline Study design & & & & & & & 0.490 \\
\hline PC-CS (A.17) & 22 & 11,524 & $\begin{array}{l}0.86(0.76 \sim \\
0.96)\end{array}$ & 0.009 & 81.0 & $<0.001$ & \\
\hline HC-CS (A.18) & 10 & 3451 & $\begin{array}{l}0.61(0.29 \sim \\
1.32)\end{array}$ & 0.214 & 96.8 & $<0.001$ & \\
\hline Cohort study (A.19) & 1 & 178 & $\begin{array}{l}1.02(0.70 \sim \\
1.48)\end{array}$ & 0.917 & NA & NA & \\
\hline Definition of reference category & & & & & & & 0.024 \\
\hline Never breastfeeding (A.20) & 25 & 13,278 & $\begin{array}{l}0.87(0.72 \sim \\
1.04)\end{array}$ & 0.132 & 93.8 & $<0.001$ & \\
\hline $\begin{array}{l}\text { Occasional breastfeeding } \\
\text { (A.21) }\end{array}$ & 8 & 1875 & $\begin{array}{l}0.47(0.28 \sim \\
0.80)\end{array}$ & 0.005 & 88.8 & $<0.001$ & 0.872 \\
\hline$\leq 6$ months (A.22) & 5 & 440 & $0.49(0.35 \sim$ & $<0.001$ & 21.7 & 0.276 & \\
\hline
\end{tabular}


Table 2 Subgroup analysis of association between breastfeeding and childhood leukemia risk for the comparison of breastfeeding versus non/occasional breastfeeding (Continued)

\begin{tabular}{|c|c|c|c|c|c|c|c|}
\hline & $\begin{array}{l}\text { No. of } \\
\text { studies }\end{array}$ & $\begin{array}{l}\text { No. of } \\
\text { cases }\end{array}$ & OR (95\%Cl) & $\begin{array}{l}P \\
\text { value }\end{array}$ & $\begin{array}{l}I^{2} \\
(\%)\end{array}$ & $\begin{array}{l}P \text { value for } \\
\text { heterogeneity }\end{array}$ & $\begin{array}{l}P \text { in meta- } \\
\text { regression }\end{array}$ \\
\hline & & & $0.67)$ & & & & \\
\hline$\leq 1$ month (A.23) & 3 & 1435 & $\begin{array}{l}0.50(0.15 \sim \\
1.60)\end{array}$ & 0.240 & 95.9 & $<0.001$ & \\
\hline
\end{tabular}

Abbreviations: PC-CS population-based case-control study; HC-CS hospital-based case-control study; NA not applicable

'One study was conducted in the United States, Canada and Australia

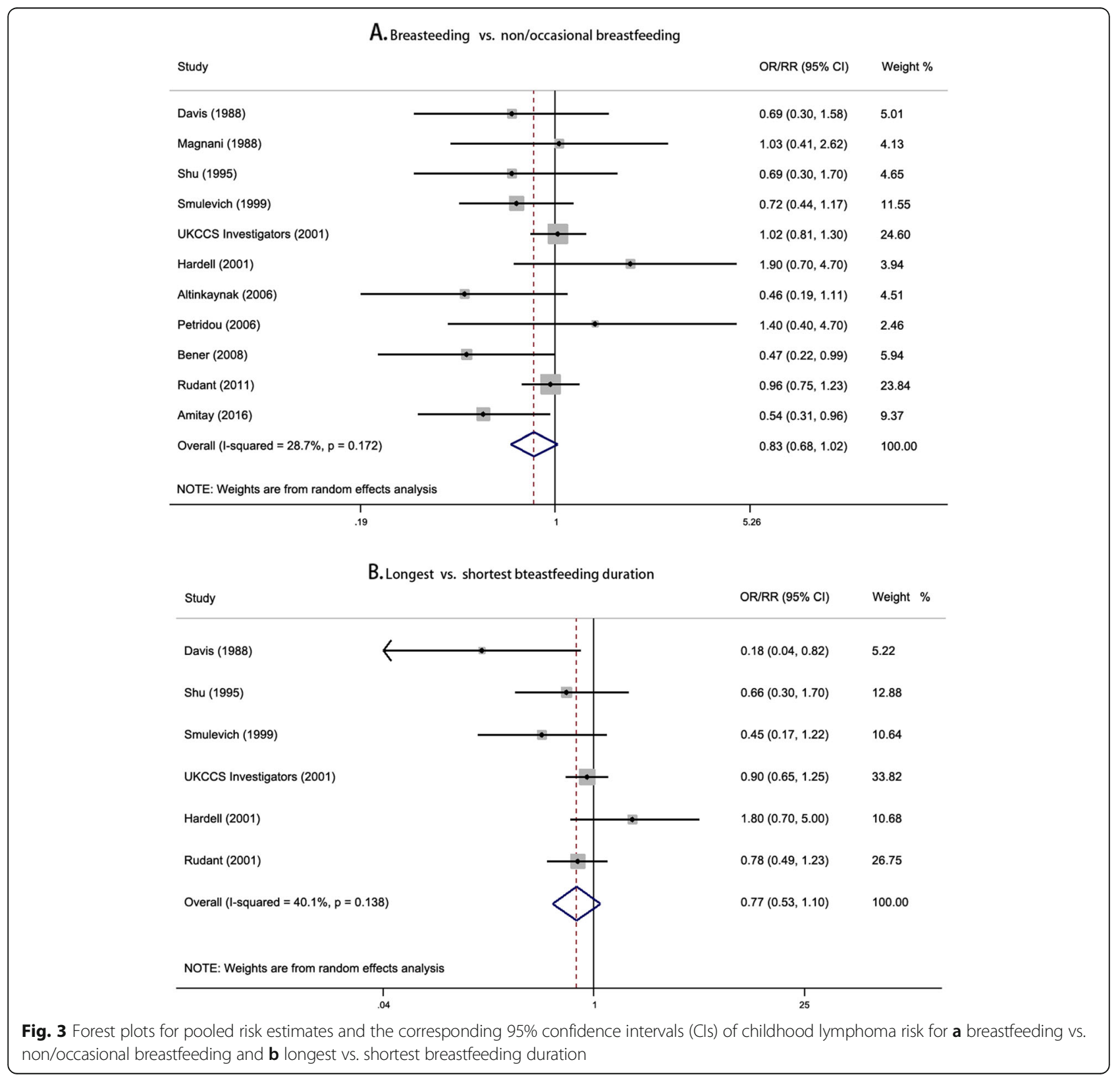


Table 3 Subgroup analysis of association between breastfeeding and childhood lymphoma risk for the comparison of breastfeeding versus non/occasional breastfeeding

\begin{tabular}{|c|c|c|c|c|c|c|c|}
\hline & $\begin{array}{l}\text { No. of } \\
\text { studies }\end{array}$ & $\begin{array}{l}\text { No. of } \\
\text { cases }\end{array}$ & OR $(95 \% \mathrm{Cl})$ & $\begin{array}{l}P \\
\text { value }\end{array}$ & $\begin{array}{l}l^{2} \\
(\%)\end{array}$ & $\begin{array}{l}P \text { value for } \\
\text { heterogeneity }\end{array}$ & $\begin{array}{l}P \text { in meta- } \\
\text { regression }\end{array}$ \\
\hline Cancer type & & & & & & & 0.875 \\
\hline Hodgkin's disease (B.1) & 9 & 458 & $\begin{array}{l}0.86(0.63 \sim \\
1.18)\end{array}$ & 0.341 & 25.8 & 0.215 & \\
\hline $\begin{array}{l}\text { Non-Hodgkin's lymphoma } \\
\text { (B.2) }\end{array}$ & 9 & 686 & $\begin{array}{l}0.88(0.67 \sim \\
1.15)\end{array}$ & 0.343 & 26.9 & 0.205 & \\
\hline Year of publication & & & & & & & 0.583 \\
\hline In and before 2000 (B.3) & 4 & 244 & $\begin{array}{l}0.75(0.53 \sim \\
1.06)\end{array}$ & 0.103 & 0.0 & 0.908 & \\
\hline After 2000 (B.4) & 7 & 980 & $\begin{array}{l}0.84(0.63 \sim \\
1.12)\end{array}$ & 0.231 & 51.5 & 0.054 & \\
\hline Geographic location & & & & & & & 0.041 \\
\hline Europe (B.5) & 6 & 939 & $\begin{array}{l}0.98(0.84 \sim \\
1.15)\end{array}$ & 0.826 & 0.0 & 0.571 & \\
\hline Asia (B.6) & 4 & 259 & $\begin{array}{l}0.53(0.37 \sim \\
0.76)\end{array}$ & 0.001 & 0.0 & 0.906 & \\
\hline North America (B.7) & 1 & 26 & $\begin{array}{l}0.69(0.30 \sim \\
1.58)\end{array}$ & 0.381 & NA & NA & \\
\hline Study quality score & & & & & & & 0.436 \\
\hline$<7$ (B.8) & 2 & 85 & $\begin{array}{l}0.66(0.31 \sim \\
1.42)\end{array}$ & 0.290 & 39.7 & 0.198 & \\
\hline$\geq 7$ (B.9) & 9 & 1139 & $\begin{array}{l}0.86(0.70 \sim \\
1.06)\end{array}$ & 0.152 & 28.0 & 0.196 & \\
\hline Sample size & & & & & & & 0.578 \\
\hline$\leq 200($ B.10) & 5 & 368 & $\begin{array}{l}0.77(0.44 \sim \\
1.35)\end{array}$ & 0.366 & 45.5 & 0.119 & \\
\hline$>200$ (B.11) & 6 & 856 & $\begin{array}{l}0.89(0.75 \sim \\
1.06)\end{array}$ & 0.203 & 12.7 & 0.334 & \\
\hline Study design & & & & & & & 0.743 \\
\hline PC-CS (B.12) & 8 & 1068 & $\begin{array}{l}0.85(0.68 \sim \\
1.05)\end{array}$ & 0.125 & 34.1 & 0.156 & \\
\hline HC-CS (B.13) & 3 & 156 & $\begin{array}{l}0.78(0.41 \sim \\
1.51)\end{array}$ & 0.461 & 31.4 & 0.233 & \\
\hline Definition of reference category & & & & & & & 0.044 \\
\hline Never breastfeeding (B.14) & 6 & 831 & $\begin{array}{l}0.97(0.83 \sim \\
1.14)\end{array}$ & 0.732 & 0.0 & 0.879 & \\
\hline $\begin{array}{l}\text { Occasional breastfeeding } \\
\text { (B.15) }\end{array}$ & 5 & 393 & $\begin{array}{l}0.66(0.44 \sim \\
0.99)\end{array}$ & 0.042 & 40.8 & 0.149 & 0.170 \\
\hline$\leq 2$ months (B.16) & 2 & 216 & $\begin{array}{l}1.07(0.42 \sim \\
2.72)\end{array}$ & 0.888 & 68.3 & 0.076 & \\
\hline$\leq 6$ months (B.17) & 3 & 177 & $\begin{array}{l}0.50(0.34 \sim \\
0.75)\end{array}$ & 0.001 & 0.0 & 0.937 & \\
\hline
\end{tabular}

Abbreviations: PC-CS population-based case-control study; HC-CS hospital-based case-control study; NA not applicable

commonly used as adjustment, because higher SES among controls may overestimate the protective effect of breastfeeding on the risk of childhood cancer [40]. Smoking during pregnancy was also considered necessary, since it is associated with breastfeeding [74], and it may be related with the risk of childhood cancer [75, 76], even though the association may vary by cancer types [77-80]. Therefore, the imbalance in this factor between cases and controls may be contributory to confounding bias. Some other potential confounders were also taken into consideration, such as infectious exposures, day care, birth weight, and birth order. However, we cannot exclude the possibility of residual confounding, although most of the included studies had matching 




and adjustment variables. In the stratified analysis of study quality, we found that the results from studies with higher quality showed consistent association with overall risk estimates, which provides additional confidence in the findings of our meta-analysis.

Case-control study is the main study design that all included studies of the meta-analysis except one were case-control studies. Since most studies have collected exposure information through parental interview, case-control studies are susceptible to recall bias and selection bias. Cohort studies are considered to provide more robust estimates than case-control studies; therefore, further cohort studies are needed to provide more evidence on the association and risk of childhood cancer. An optimal study might be conducted within the framework of a large populationbased registry or cohort with full access to baseline information regarding demographic characteristics, detailed data of breastfeeding including breastfeeding duration, the use of infant formulas, the main types of milk given, the age of introduction of a range of foods and so on, and collection of medical records to accurately identify all diagnosed cases [81]. However, cohort studies would require follow-up periods of several years consuming manpower, material, financial and time largely, and very large sample sizes to provide sufficient statistical power. To be noted, the findings are to be expected from International Childhood Cancer Consortium (I4C), which is the first cohort consortium to have published findings on childhood cancer to elaborate the association [82]. But for now, case-control studies are mainly reported, and results from population-based case-control studies are more reliable compared with those from hospital-based 
Table 4 Subgroup analysis of association between breastfeeding and risk of childhood brain tumors for the comparison of breastfeeding versus non/occasional breastfeeding

\begin{tabular}{|c|c|c|c|c|c|c|c|}
\hline & $\begin{array}{l}\text { No. of } \\
\text { studies }\end{array}$ & $\begin{array}{l}\text { No. of } \\
\text { cases }\end{array}$ & OR $(95 \% \mathrm{Cl})$ & $\begin{array}{l}P \\
\text { value }\end{array}$ & $\begin{array}{l}I^{2} \\
(\%)\end{array}$ & $\begin{array}{l}P \text { value for } \\
\text { heterogeneity }\end{array}$ & $\begin{array}{l}P \text { in meta- } \\
\text { regression }\end{array}$ \\
\hline Cancer type & & & & & & & 0.396 \\
\hline Ependymoma (C.1) & 4 & 408 & $\begin{array}{l}1.02(0.65 \sim \\
1.60)\end{array}$ & 0.931 & 22.3 & 0.277 & \\
\hline Astrocytoma (C.2) & 4 & 475 & $\begin{array}{l}0.91(0.71 \sim \\
1.15)\end{array}$ & 0.411 & 0.0 & 0.589 & \\
\hline Medulloblastoma (C.3) & 2 & 148 & $\begin{array}{l}0.89(0.42 \sim \\
1.92)\end{array}$ & 0.770 & 53.4 & 0.143 & \\
\hline Others (C.4) & 3 & 446 & $\begin{array}{l}1.13(0.83 \sim \\
1.55)\end{array}$ & 0.446 & 22.1 & 0.274 & \\
\hline Year of publication & & & & & & & 0.665 \\
\hline In and before 2005 (C.5) & 4 & 640 & $\begin{array}{l}0.95(0.69 \sim \\
1.30)\end{array}$ & 0.751 & 37.8 & 0.186 & \\
\hline After 2005 (C.6) & 3 & 1019 & $\begin{array}{l}0.94(0.80 \sim \\
1.10)\end{array}$ & 0.431 & 0.0 & 0.458 & \\
\hline Geographic location & & & & & & & 0.296 \\
\hline North America (C.7) & 2 & 310 & $\begin{array}{l}0.74(0.55 \sim \\
1.01)\end{array}$ & 0.055 & 0.0 & 0.364 & \\
\hline Europe (C.8) & 4 & 1071 & $\begin{array}{l}1.03(0.90 \sim \\
1.19)\end{array}$ & 0.639 & 0.0 & 0.636 & \\
\hline Oceania (C.9) & 1 & 278 & $\begin{array}{l}0.81(0.47 \sim \\
1.39)\end{array}$ & 0.443 & NA & NA & \\
\hline Study quality score & & & & & & & 0.847 \\
\hline$\leq 7(C .10)$ & 5 & 926 & $\begin{array}{l}0.92(0.72 \sim \\
1.16)\end{array}$ & 0.472 & 35.6 & 0.184 & \\
\hline$>7$ (C.11) & 2 & 733 & $\begin{array}{l}0.98(0.82 \sim \\
1.17)\end{array}$ & 0.825 & 0.0 & 0.479 & \\
\hline Sample size & & & & & & & 0.179 \\
\hline$\leq 1000(C .12)$ & 4 & 631 & $\begin{array}{l}0.81(0.62 \sim \\
1.06)\end{array}$ & 0.121 & 4.7 & 0.369 & \\
\hline$>1000(C .13)$ & 3 & 1028 & $\begin{array}{l}1.02(0.89 \sim \\
1.17)\end{array}$ & 0.772 & 0.0 & 0.560 & \\
\hline Definition of reference category & & & & & & & 0.665 \\
\hline Never breastfeeding (C.14) & 3 & 1019 & $\begin{array}{l}0.94(0.80 \sim \\
1.10)\end{array}$ & 0.431 & 0.0 & 0.458 & \\
\hline $\begin{array}{l}\text { Occasional breastfeeding } \\
\text { (C.15) }\end{array}$ & 4 & 640 & $\begin{array}{l}0.95(0.69 \sim \\
1.30)\end{array}$ & 0.751 & 37.8 & 0.186 & 0.207 \\
\hline$\leq 2$ months $(C .16)$ & 3 & 602 & $\begin{array}{l}1.08(0.88 \sim \\
1.32)\end{array}$ & 0.482 & 0.0 & 0.489 & \\
\hline$\leq 6$ months $(C .17)$ & 1 & 38 & $\begin{array}{l}0.57(0.30 \sim \\
1.09)\end{array}$ & 0.088 & NA & NA & \\
\hline
\end{tabular}

Abbreviations: PC-CS population-based case-control study; HC-CS hospital-based case-control study; NA not applicable

case-control studies. We also found similar association in population-based case-control studies with overall risk estimates.

\section{Leukemia}

There is sufficient evidence to show that breastfeeding was inversely associated with the risk by pooling a number of original articles, with $23 \%$ lower risk of childhood leukemia (95\% CI 9-35\%) for breastfeeding versus non/ occasional breastfeeding and 23\% lower risk (95\% CI 6$37 \%)$ for longest versus shortest breastfeeding duration, respectively. Moreover, we found a protective effect of breastfeeding on the risk of childhood ALL among the studies with higher quality and population-based casecontrol studies.

We found high heterogeneity in the meta-analysis on the association between breastfeeding and the risk of childhood leukemia, and there exists significant 
Table 5 Numbers of retrieved studies and pooled odds ratio for the association of breastfeeding with the risk of five individual cancer types

\begin{tabular}{|c|c|c|c|c|c|c|c|c|c|c|}
\hline \multirow[b]{2}{*}{ Cancer type } & \multicolumn{5}{|c|}{ Ever versus non/occasional breastfeeding } & \multicolumn{5}{|c|}{ Longest breastfeeding versus shortest breastfeeding } \\
\hline & $\begin{array}{l}\text { No. of } \\
\text { studies }\end{array}$ & OR $(95 \% \mathrm{Cl})$ & $\begin{array}{l}P \\
\text { value }\end{array}$ & $\begin{array}{l}l^{2} \\
(\%)\end{array}$ & $\begin{array}{l}P \text { value for } \\
\text { heterogeneity }\end{array}$ & $\begin{array}{l}\text { No. of } \\
\text { studies }\end{array}$ & OR $(95 \% \mathrm{Cl})$ & $\begin{array}{l}P \\
\text { value }\end{array}$ & $\begin{array}{l}l^{2} \\
\text { (\%) }\end{array}$ & $\begin{array}{l}P \text { value for } \\
\text { heterogeneity }\end{array}$ \\
\hline Neuroblastoma & 4 & $\begin{array}{l}0.59(0.44 \sim \\
0.81)\end{array}$ & 0.001 & 29.2 & 0.237 & 4 & $\begin{array}{l}0.61(0.44 \sim ~ \\
0.83)\end{array}$ & 0.002 & 0.0 & 0.482 \\
\hline $\begin{array}{l}\text { Soft-tissue } \\
\text { sarcoma }\end{array}$ & 4 & $\begin{array}{l}0.79(0.44 \sim \\
1.44)\end{array}$ & 0.448 & 37.1 & 0.189 & 3 & $\begin{array}{l}0.69(0.14 \sim \\
3.44)\end{array}$ & 0.649 & 63.9 & 0.063 \\
\hline Nephroblastoma & 3 & $\begin{array}{l}0.63(0.41 \sim \\
0.98)\end{array}$ & 0.041 & 57.2 & 0.097 & 3 & $\begin{array}{l}0.76(0.44 \sim \\
1.32)\end{array}$ & 0.324 & 49.1 & 0.140 \\
\hline Retinoblastoma & 2 & $\begin{array}{l}1.32(0.17 \sim \\
10.24)\end{array}$ & 0.792 & 72.6 & 0.056 & 2 & $\begin{array}{l}1.08(0.57 \sim \\
2.07)\end{array}$ & 0.807 & 0.0 & 0.411 \\
\hline $\begin{array}{l}\text { Germ cell } \\
\text { tumors }\end{array}$ & 2 & $\begin{array}{l}1.08(0.73 \sim \\
1.60)\end{array}$ & 0.708 & 0.0 & 0.639 & 2 & $\begin{array}{l}2.64(1.05 \sim ~ \\
6.60)\end{array}$ & 0.038 & 0.0 & 0.319 \\
\hline
\end{tabular}

heterogeneity in different regional groups by meta-regression analysis. In the subgroup analysis of geographic location, we found that breastfeeding was more strongly associated with the risk of childhood leukemia in Asia. In terms of risk of bias, Robins-I tool rated 4 European studies and 2 Asian studies at serious risk, and 10 European studies and 6 Asian studies were considered at moderate risk. At the original study level, one aspect that deserves particular attention is the difference in the number of cases and the source of population, which may at least in part explain the heterogeneity observed across the geographic region. For example, the Asian subgroup analyses included 8 studies with a total of 1739 cases, with only 1 study enrolled more than 200 cases. Moreover, few studies (3/8) used population-based case-control study design. However, European articles included 14 studies with 7518 cases and $71 \%$ studies used population controls. This is a similar situation to North America or Oceania, in that a majority (6/11) of articles included in the meta-analysis had more than 200 case numbers, and most (9/11) used population controls. Therefore, the differences in risk estimates could be related to study quality issues. On the other hand, there were several other potential explanations could be proposed. A great variation in breastfeeding duration across the countries may result in the heterogeneity. It should be noted that breastfeeding duration is shorter in highincome countries than in those that are resource-poor. It was estimated that $25 \%$ of infants in the USA and Europe are exclusively breastfed through 6 months [83, 84], as compared with $43 \%$ in the South-East Asia region [85]. In particular, only three countries (France, Spain, and the USA) had rates below $80 \%$ for ever breastfeeding across all country groups [10]. It could be that varying cultural influences contribute to this region disparity, with for example protective Islamic beliefs, South Asian cultural teachings, and more extensive support networks. A study in UK provided the evidence that Pakistan- origin mothers had higher breastfeeding initiation rates and longer average breastfeeding durations than White British mothers [86]. Another cross-sectional study in the USA demonstrated that Asian women had the highest breastfeeding initiation rates relative to all other racial/ethnic groups [87]. On the other hand, the heterogeneity may also due to the breastfeeding pattern. The volume of breastfeeding differed considerably between the women who breastfed only and those who performed mixed feeding, even if these women had the same breastfeeding duration. Globally, the prevalence of exclusive breastfeeding varied widely, countries from Asia and the Pacific region had moderate to high rate of exclusive breastfeeding, while the rate of exclusive breastfeeding was lower in Europe and America [10].

Additionally, we performed the dose-response metaanalysis, which showed a specific non-linear doseresponse relationship between breastfeeding and the risk of childhood leukemia. World Health Organization (WHO) and United Nations International Children's Emergency Fund (UNICEF) developed the global strategy for infant and young child feeding that infants should be exclusively breastfed for the first 6 months of life to achieve optimal growth, development and health [88]. In the current study, we found that breastfeeding duration of 6 months could reduce 20\% (95\% CI 15$25 \%)$ risk of childhood leukemia. The U-shaped curve showed that breastfeeding duration of approximately 9.6 months might show the most significant protect effect on the risk of childhood leukemia. The decreased risk of childhood leukemia was statistically significant at a duration of 4.4-15.0 months. Interestingly, we found that there was more pronounced association between breastfeeding and risk of childhood leukemia when defining breastfeeding for less than 6 months as reference group, rather than never breastfeeding, which may due to that breastfeeding appeared a protective effect on the risk of leukemia after a certain period. The nonsignificant 
decreased risk at prolonged breastfeeding duration might derive from relatively small sample size but not real effect. Large-sample and well-designed studies should be developed in future to demonstrate this turning point.

There were several potential explanations why breastfeeding may decrease the risk of childhood leukemia. Breast milk contains high levels of immunologically active components and multifactorial anti-inflammatory defense mechanisms that influence the development of the immune system of the breastfed infant $[89,90]$. For example, soluble tumor necrosis factor (TNF)-related apoptosis-inducing ligand (TRAIL) in breast milk can control apoptosis and cell proliferation in various organs and tissues. Breastfeeding also provides the infants with human alpha-lactalbumin made lethal to tumor cells (HAMLET), which is a substance with anticancer activity in breast milk [91]. Besides, breast milk imparts the mother's stem cells to the infant, where they potentially function to actively stimulate or modulate the immune system and promote its development early in life [92]. In particular, Greaves' hypothesis proposed immunological model that breast milk could modify the immune response in the prevention of childhood ALL [93]. Moreover, accumulating evidence have demonstrated that breast milk has the potential of shaping the neonate's gut microbiome, such as microbiota richness, diversity, and composition [94, 95]. Recently, a large, multi-center study suggested that breastfeeding status was the most significant factor associated with microbiome structure in early life [96]. It is hypothesized that breastfeeding could decrease the risk of childhood leukemia by the recent discovery of the breast milk microbiome and its connections with immune factors [97].

\section{Lymphoma}

The meta-analysis provided no convincing evidence of the association between breastfeeding and risk of lymphoma. The findings of the present study are consistent with previous observational studies although some early studies indicated negative association of breastfeeding with lymphoma risk, especially in Hodgkin's lymphoma [19, 43]. In addition, results from sensitivity analysis were unstable, which deserves more studies to clarify the association.

Our pooled analysis presented an inverse association between breastfeeding and risk of childhood lymphoma in Asia, but not Europe or North America, suggesting a potential region-specific effect. What is more, significant discrepancies across subgroups stratified by definition of reference category were found, and only the pooled estimate for the studies using occasional breastfeeding as reference group showed a significantly decreased risk of childhood lymphoma. It may be explained by the significant non-linear dose-response relationship. For example, Davis et al. [33] found that breastfeeding for more than
6 months was associated with a decreased risk of childhood lymphoma, but that breastfeeding for shorter durations was not associated with a reduced risk. The study using breastfeeding for less than 6 months as reference group suggested that a longer breastfeeding duration had a protective effect against Hodgkin's lymphoma [53]. Another point we should make is that the incidence of lymphoma is highest among 10-14 years old (and even higher among 15-19 years old), while the low age of the cases in the included studies with a short tumor induction period might be too short to find a decreased risk for lymphoma.

\section{Other cancers}

We found significant association between breastfeeding and risk of childhood neuroblastoma, while no significant associations of breastfeeding with risk of soft-tissue sarcoma, brain tumors, nephroblastoma, retinoblastoma, and germ cell tumors. This is updated from the previous meta-analysis and the results were consistent [19]. However, the associations of breastfeeding and risk of these cancers may be underpowered because of the small number of studies in the meta-analysis.

\section{Strength and limitation}

The primary strength of our study is that the traditional categorical meta-analysis and dose-response analysis were applied simultaneously, which can provide more meaningful information. Another strength is the large sample size and number of included studies, which make the findings stable and reliable and enable us to conduct multiple subgroup analyses by geographic location, study quality score, study design, etc. In addition, we also performed stratified analyses on the association of breastfeeding and risk of the subtypes of individual cancers.

However, the present study has several limitations. First, there was high evidence of heterogeneity across studies in the categorical meta-analysis. However, metaregression analyses suggested that geographic region and definition of reference category are the potential sources of the observed heterogeneity. Second, our meta-analysis included very limited studies from Oceania and no study from Africa. Third, the results of dose-response metaanalysis were prone to be influenced by possible exposure misclassification as the exposure dose was estimated with median for interval exposure, and the lower bound added to the half of the adjacent previous category for the highest open-ended exposure group. Fourth, the number of studies evaluating the associations of breastfeeding with risk of neuroblastoma, nephroblastoma, retinoblastoma, and germ cell tumors is small. Fifth, we were unable to assess differences by age or sex, because sufficiently age- or sex-specific studies are not available. Finally, limited by the lack information of breastfeeding pattern in most of included studies, such as exclusive 
breastfeeding and partial breastfeeding, we cannot evaluate the association between breastfeeding pattern and the risk of childhood cancer.

\section{Conclusion}

This meta-analysis demonstrates that breastfeeding was associated with the reduced the risk of childhood leukemia. The present study also provides suggestive evidence of the inverse association between breastfeeding and risk of neuroblastoma. In addition, given that the role of breastfeeding for the risk of childhood leukemia and lymphoma may be region-specific, further analyses are warranted to provide insights into the strategy of breastfeeding advocacy.

\section{Abbreviations}

Cl: Confidence interval; HAMLET: Human alpha-lactalbumin made lethal to tumor cells; OR: Odds ratio; PRISMA: Preferred Reporting Items for Systematic reviews and Meta-Analysis; RR: Relative risk; TNF: Tumor necrosis factor; TRAI L: TNF-related apoptosis-inducing ligand; UNICEF: United Nations International Children's Emergency Fund; WHO: World Health Organization

\section{Supplementary Information}

The online version contains supplementary material available at https://doi. org/10.1186/s12916-021-01950-5.

Additional file 1: Table S1. The PRISMA 2009 checklist for this metaanalysis. Figure S1. Flowchart of study selection. Table S2. List of excluded studies along with reason. Table S3. Details of included studies for subgroup analysis. Figure S2. Begg's funnel plots identifying the publication bias for the association between breastfeeding and risk of childhood leukemia. Figure S3. Forest plots of subgroup analysis of association between breastfeeding and childhood leukemia risk in the order listed in Table 2. Table S4. Subgroup analyses of the association between breastfeeding and acute lymphoblastic leukemia risk. Figure S4. One-study-removed analysis on the association of breastfeeding with risk of (A) childhood leukemia and (B) acute lymphoblastic leukemia. Figure S5. Pooled analysis of studies including only children aged 0-14 years old for the for the association of breastfeeding with risk of (A) childhood leukemia and (B) acute lymphoblastic leukemia. Figure S6. Risk estimates (solid line) and the corresponding 95\% Cls (dash lines) for the dose-response relationship between breastfeeding and the risk of childhood lymphoma. Figure S7. Begg's funnel plots identifying the publication bias for the association between breastfeeding and risk of childhood lymphoma. Figure S8. Forest plots of subgroup analysis of association between breastfeeding and childhood lymphoma risk in the order listed in Table 3. Figure S9. One-study-removed analysis on the association of breastfeeding with risk of childhood lymphoma. Figure S10. Pooled analysis of studies including only children aged 0-14 years old for the for the association of breastfeeding with risk of childhood lymphoma. Figure S11. Begg's funnel plots identifying the publication bias for the association between breastfeeding and risk of childhood brain tumors. Figure S12. Forest plots of subgroup analysis of association between breastfeeding and risk of childhood brain tumors in the order listed in Table 4. Figure S13. One-study-removed analysis on the association of breastfeeding with risk of childhood brain tumors. Figure S14. Pooled analysis of studies including only children aged $0-14$ years old for the for the association of breastfeeding with risk of childhood brain tumors.

\section{Acknowledgements}

The authors thank all the participants and researchers of the studies included for the meta-analysis.

\section{Authors' contributions}

DY and YM conceived the study. QS and QY searched the databases and checked them according to the eligible criteria and exclusion criteria. XS and PZ helped develop search strategies. DY, QS, XS, and LZ analyzed the data and wrote the draft of the paper. LZ, YM, and DY contributed to reviewing or revising the paper. DY and YM are the guarantors of this work. All authors read and approve the final manuscript.

\section{Funding}

This study was funded by the National Natural Science Foundation of China (No. 81973663), Natural Science Foundation of Zhejiang Province (No. LQ20H260008), and Medical Health Science and Technology Project of Zhejiang Provincial Health Commission (2020KY195).

Availability of data and materials

All data generated or analyzed during this study are included in this article and its additional files.

\section{Declarations}

Ethics approval and consent to participate

Not applicable.

\section{Consent for publication}

Not applicable.

\section{Competing interests}

The authors have declared no conflict of interests.

\section{Author details}

${ }^{1}$ Department of Epidemiology and Biostatistics, School of Public Health, Zhejiang Chinese Medical University, 548 Binwen Road, Hangzhou 310053, China. ${ }^{2}$ Department of Hematology and Oncology, Children's hospital of Nanjing Medical University, Nanjing 210008, China. ${ }^{3}$ Department of Medical Adiministration, Women's Hospital, Zhejiang University School of Medicine, Hangzhou 310006, China.

Received: 7 October 2020 Accepted: 24 February 2021

Published online: 13 April 2021

\section{References}

1. Lam CG, Howard SC, Bouffet E, et al. Science and health for all children with cancer. Science. 2019;363:1182-6.

2. Bray F, Ferlay J, Soerjomataram I, et al. Global cancer statistics 2018: GLOBOCAN estimates of incidence and mortality worldwide for 36 cancers in 185 countries. CA Cancer J Clin. 2018;68:394-424.

3. Steliarova-Foucher $E$, Colombet $M$, Ries $L$, et al. International incidence of childhood cancer, 2001-10: a population-based registry study. Lancet Oncol. 2017;18:719-31.

4. Belson M, Kingsley B, Holmes A. Risk factors for acute leukemia in children: a review. Environ Health Perspect. 2007;115:138-45.

5. Wigle DT, Arbuckle TE, Turner MC, et al. Epidemiologic evidence of relationships between reproductive and child health outcomes and environmental chemical contaminants. J Toxicol Environ Health B Crit Rev. 2008;11:373-517.

6. Spector LG, Pankratz N, Marcotte EL. Genetic and nongenetic risk factors for childhood cancer. Pediatr Clin N Am. 2015;62:11-25.

7. Islami F, Liu Y, Jemal A, et al. Breastfeeding and breast cancer risk by receptor status--a systematic review and meta-analysis. Ann Oncol. 2015;26: 2398-407.

8. Luan NN, Wu QJ, Gong TT, et al. Breastfeeding and ovarian cancer risk: a meta-analysis of epidemiologic studies. Am J Clin Nutr. 2013;98:1020-31.

9. Gartner LM, Morton J, Lawrence RA, et al. Breastfeeding and the use of human milk. Pediatrics. 2005:115:496-506.

10. Victora CG, Bahl R, Barros AJ, et al. Breastfeeding in the 21st century: epidemiology, mechanisms, and lifelong effect. Lancet. 2016;387:475-90.

11. Kucukcongar A, Oguz A, Pinarli FG, et al. Breastfeeding and childhood cancer: is breastfeeding preventative to childhood cancer? Pediatr Hematol Oncol. 2015;32:374-81.

12. Ortega-Garcia JA, Ferris-Tortajada J, Torres-Cantero AM, et al. Full breastfeeding and paediatric cancer. J Paediatr Child Health. 2008;44:10-3. 
13. Lancashire RJ, Sorahan T. Breastfeeding and childhood cancer risks: OSCC data. Br J Cancer. 2003;88:1035-7.

14. Hardell L, Dreifaldt AC. Breast-feeding duration and the risk of malignant diseases in childhood in Sweden. Eur J Clin Nutr. 2001;55:179-85.

15. Amitay EL, Keinan-Boker L. Breastfeeding and childhood leukemia incidence: A Meta-analysis and systematic review. JAMA Pediatr. 2015;169(6):e151025.

16. Wang KL, Liu CL, Zhuang Y, et al. Breastfeeding and the risk of childhood Hodgkin lymphoma: a systematic review and meta-analysis. Asian Pac J Cancer Prev. 2013;14(8):4733-7.

17. Shu XO, Nesbit ME, Buckley JD, et al. An exploratory analysis of risk factors for childhood malignant germ-cell tumors: report from the Childrens Cancer Group (Canada, United States). Cancer Causes Control. 1995;6:18798.

18. Jimenez-Hernandez E, Fajardo-Gutierrez A, Nunez-Enriquez JC, et al. A greater birthweight increases the risk of acute leukemias in Mexican children-experience from the Mexican Interinstitutional Group for the identification of the causes of childhood leukemia (MIGICCL). Cancer Med. 2018;7:1528-36.

19. Martin RM, Gunnell D, Owen CG, et al. Breast-feeding and childhood cancer: a systematic review with metaanalysis. Int J Cancer. 2005;117:1020-31.

20. Rudant J, Lightfoot T, Urayama KY, et al. Childhood acute lymphoblastic leukemia and indicators of early immune stimulation: a Childhood Leukemia International Consortium study. Am J Epidemiol. 2015;181(8):549-62.

21. Birch JM, Marsden HB. A classification scheme for childhood cancer. Int J Cancer. 1987;40(5):620-4.

22. Kramárová E, Stiller CA. The international classification of childhood cancer. Int J Cancer. 1996;68(6):759-65.

23. Steliarova-Foucher E, Stiller C, Lacour B, et al. International classification of childhood cancer, third edition. Cancer. 2005;103(7):1457-67.

24. Stang A. Critical evaluation of the Newcastle-Ottawa scale for the assessment of the quality of nonrandomized studies in meta-analyses. Eur J Epidemiol. 2010;25:603-5.

25. Sterne JA, Hernán MA, Reeves BC, et al. ROBINS-I: a tool for assessing risk of bias in non-randomised studies of interventions. BMJ. 2016;355:14919.

26. Orsini N. From floated to conventional confidence intervals for the relative risks based on published dose-response data. Comput Methods Prog Biomed. 2010;98:90-3.

27. Orsini $N$, Li R, Wolk A, et al. Meta-analysis for linear and nonlinear doseresponse relations: examples, an evaluation of approximations, and software. Am J Epidemiol. 2012;175:66-73.

28. Higgins JP, Thompson SG, Deeks JJ, et al. Measuring inconsistency in metaanalyses. BMJ. 2003;327:557-60

29. Chen B, Benedetti A. Quantifying heterogeneity in individual participant data meta-analysis with binary outcomes. Syst Rev. 2017;6:243.

30. DerSimonian R, Laird N. Meta-analysis in clinical trials. Control Clin Trials. 1986;7:177-88

31. Hayashino Y, Noguchi Y, Fukui T. Systematic evaluation and comparison of statistical tests for publication bias. J Epidemiol. 2005;15:235-43.

32. Begg CB, Mazumdar M. Operating characteristics of a rank correlation test for publication bias. Biometrics. 1994;50:1088-101.

33. Davis MK, Savitz DA, Graubard BI. Infant feeding and childhood cancer. Lancet. 1988;2:365-8.

34. Magnani C, Pastore G, Terracini B. Infant feeding and childhood cancer. Lancet. 1988;2:1136.

35. van Duijn $\mathrm{CM}$, van Steensel-Moll HA, van der Does-vd BA, et al. Infant feeding and childhood cancer. Lancet. 1988;2:796-7.

36. Shu XO, Clemens J, Zheng W, et al. Infant breastfeeding and the risk of childhood lymphoma and leukaemia. Int J Epidemiol. 1995;24:27-32.

37. Petridou E, Trichopoulos D, Kalapothaki V, et al. The risk profile of childhood leukaemia in Greece: a nationwide case-control study. Br J Cancer. 1997;76:1241-7.

38. Schuz J, Kaletsch U, Meinert R, et al. Association of childhood leukaemia with factors related to the immune system. Br J Cancer. 1999:80:585-90.

39. Shu XO, Linet MS, Steinbuch M, et al. Breast-feeding and risk of childhood acute leukemia. J Natl Cancer Inst. 1999:91:1765-72.

40. Smulevich VB, Solionova LG, Belyakova SV. Parental occupation and other factors and cancer risk in children: I. Study methodology and nonoccupational factors. Int J Cancer. 1999;83:712-7.

41. Dockerty JD, Skegg DC, Elwood JM, et al. Infections, vaccinations, and the risk of childhood leukaemia. Br J Cancer. 1999;80:1483-9.

42. Infante-Rivard C, Fortier I, Olson E. Markers of infection, breast-feeding and childhood acute lymphoblastic leukaemia. Br J Cancer. 2000;83:1559-64.
43. UK Childhood Cancer Study Investigators. Breastfeeding and childhood cancer. Br J Cancer. 2001:85:1685-94.

44. Schuz J, Kaletsch U, Kaatsch P, et al. Risk factors for pediatric tumors of the central nervous system: results from a German population-based casecontrol study. Med Pediatr Oncol. 2001;36:274-82.

45. Daniels JL, Olshan AF, Pollock BH, et al. Breast-feeding and neuroblastoma, USA and Canada. Cancer Causes Control. 2002;13:401-5.

46. Murray L, McCarron P, Bailie K, et al. Association of early life factors and acute lymphoblastic leukaemia in childhood: historical cohort study. Br J Cancer. 2002;86:356-61.

47. Perrillat F, Clavel J, Jaussent I, et al. Breast-feeding, fetal loss and childhood acute leukaemia. Eur J Pediatr. 2002;161:235-7.

48. Jourdan-Da SN, Perel $Y$, Mechinaud F, et al. Infectious diseases in the first year of life, perinatal characteristics and childhood acute leukaemia. Br J Cancer. 2004:90:139-45.

49. Altinkaynak S, Selimoglu MA, Turgut A, et al. Breast-feeding duration and childhood acute leukemia and lymphomas in a sample of Turkish children. Pediatr Gastroenterol Nutr. 2006;42:568-72.

50. Saddlemire S, Olshan AF, Daniels JL, et al. Breast-feeding and Wilms tumor: a report from the Children's Oncology Group. Cancer Causes Control. 2006; 17:687-93.

51. Shaw AK, Li P, Infante-Rivard C. Early infection and risk of childhood brain tumors (Canada). Cancer Causes Control. 2006;17:1267-74.

52. Petridou $\mathrm{E}$, Andrie $\mathrm{E}$, Dessypris $\mathrm{N}$, et al. Incidence and characteristics of childhood Hodgkin's lymphoma in Greece: a nationwide study (Greece). Cancer Causes Control. 2006;17:209-15.

53. Bener A, Hoffmann GF, Afify Z, et al. Does prolonged breastfeeding reduce the risk for childhood leukemia and lymphomas? Minerva Pediatr. 2008;60: 155-61.

54. MacArthur AC, McBride ML, Spinelli JJ, et al. Risk of childhood leukemia associated with vaccination, infection, and medication use in childhood: the cross-Canada childhood leukemia study. Am J Epidemiol. 2008:167:598-606.

55. Flores-Lujano J, Perez-Saldivar ML, Fuentes-Panana EM, et al. Breastfeeding and early infection in the aetiology of childhood leukaemia in Down syndrome. Br J Cancer. 2009;101:860-4.

56. Rudant J, Orsi L, Menegaux F, et al. Childhood acute leukemia, early common infections, and allergy: the ESCALE study. Am J Epidemiol. 2010; 172:1015-27.

57. Rudant J, Orsi L, Monnereau A, et al. Childhood Hodgkin's lymphoma, nonHodgkin's lymphoma and factors related to the immune system: the Escale study (SFCE). Int J Cancer. 2011;129:2236-47.

58. Waly Ml, Ali A, Al-Saadoon M, et al. Breastfeeding is not associated with risk of developing childhood leukemia in the Sultanate of Oman. Asian Pac J Cancer Prev. 2011;12:2087-91.

59. Urayama KY, Chokkalingam AP, Metayer C, et al. HLA-DP genetic variation, proxies for early life immune modulation and childhood acute lymphoblastic leukemia risk. Blood. 2012;120:3039-47.

60. Lupo PJ, Zhou R, Skapek SX, et al. Allergies, atopy, immune-related factors and childhood rhabdomyosarcoma: a report from the Children's Oncology Group. Int J Cancer. 2014;134:431-6.

61. Mattioli S, Farioli A, Legittimo P, et al. Tobacco smoke and risk of childhood acute non-lymphocytic leukemia: findings from the SETIL study. PLoS One. 2014;9:e111028.

62. Schraw JM, Dong YQ, Okcu MF, et al. Do longer formula feeding and later introduction of solids increase risk for pediatric acute lymphoblastic leukemia? Cancer Causes Control. 2014;25:73-80.

63. Ajrouche R, Rudant J, Orsi L, et al. Childhood acute lymphoblastic leukaemia and indicators of early immune stimulation: the Estelle study (SFCE). Br J Cancer. 2015;112:1017-26.

64. Greenop KR, Bailey HD, Miller M, et al. Breastfeeding and nutrition to 2 years of age and risk of childhood acute lymphoblastic leukemia and brain tumors. Nutr Cancer. 2015:67:431-41.

65. Heck JE, Omidakhsh N, Azary S, et al. A case-control study of sporadic retinoblastoma in relation to maternal health conditions and reproductive factors: a report from the Children's Oncology group. BMC Cancer. 2015;15: 735.

66. Amitay EL, Dubnov RG, Keinan-Boker L. Breastfeeding, other early life exposures and childhood leukemia and lymphoma. Nutr Cancer. 2016;68: 968-77.

67. Rios $P$, Bailey $H D$, Orsi $L$, et al. Risk of neuroblastoma, birth-related characteristics, congenital malformations and perinatal exposures: a pooled 
analysis of the ESCALE and ESTELLE French studies (SFCE). Int J Cancer. 2016;139:1936-48.

68. Bailey HD, Rios P, Lacour B, et al. Factors related to pregnancy and birth and the risk of childhood brain tumours: the ESTELLE and ESCALE studies (SFCE, France). Int J Cancer. 2017;140:1757-69.

69. Schraw JM, Scheurer ME, Forman MR. A vulnerable age for the introduction of solid foods in pediatric acute lymphoblastic leukemia. Nutr Cancer. 2017; 69:261-6.

70. Gao Z, Wang R, Qin ZX, et al. Protective effect of breastfeeding against childhood leukemia in Zhejiang Province, P. R. China: a retrospective casecontrol study. Libyan J Med. 2018;13:1508273.

71. Lingappa ALKSRS. Breastfeeding and its associated risk in children with acute leukemia: a retrospective study. Indian J Med Paediatr Oncol. 2018;39: $312-5$.

72. Rafieemehr $\mathrm{H}$, Calhor F, Esfahani H, et al. Risk of acute lymphoblastic leukemia: results of a case-cntrol study. Asian Pac J Cancer Prev. 2019;20: 2477-83.

73. Bauer $H$, Rios $P$, Schleiermacher $G$, et al. Maternal and perinatal characteristics, congenital malformations and the risk of wilms tumor: the ESTELLE study. Cancer Causes Control. 2020;31:491-501.

74. Cohen SS, Alexander DD, Krebs NF, et al. Factors associated with breastfeeding initiation and continuation: a meta-analysis. J Pediatr. 2018; 203:190-6.

75. Stjernfeldt M, Berglund K, Lindsten J, et al. Maternal smoking during pregnancy and risk of childhood cancer. Lancet. 1986; (8494):1350-2.

76. Rumrich IK, Viluksela M, Vahakangas $K$, et al. Maternal smoking and the risk of cancer in early life - a meta-analysis. PLoS One. 2016;11(11):e0165040.

77. Antonopoulos CN, Sergentanis TN, Papadopoulou C, et al. Maternal smoking during pregnancy and childhood lymphoma: a meta-analysis. Int J Cancer. 2011;129(11):2694-703.

78. Chu P, Wang H, Han S, et al. Maternal smoking during pregnancy and risk of childhood neuroblastoma: systematic review and meta-analysis. J Cancer Res Ther. 2016;12(2):999-1005.

79. Bailey HD, Lacour B, Guerrini-Rousseau L, et al. Parental smoking, maternal alcohol, coffee and tea consumption and the risk of childhood brain tumours: the ESTELLE and ESCALE studies (SFCE, France). Cancer Causes Control. 2017;28:719-32.

80. Metayer C, Petridou E, Arangure JM, et al. Parental tobacco smoking and acute myeloid leukemia: The Childhood Leukemia International Consortium. Am J Epidemiol. 2016;184(4):261-73.

81. Guise $J M$, Austin $D$, Morris CD. Review of case-control studies related to breastfeeding and reduced risk of childhood leukemia. Pediatrics. 2005;116: e724-31.

82. Tikellis G, Dwyer T, Paltiel O, et al. The International Childhood Cancer Cohort Consortium (I4C): a research platform of prospective cohorts for studying the aetiology of childhood cancers. Paediatr Perinat Epidemiol. 2018;32(6):568-83.

83. Division of Nutrition, Physical Activity, and Obesity, National Center for Chronic Disease Prevention and Health Promotion. Breastfeeding Report Card: United States. 2020. https://www.cdc.gov/breastfeeding/pdf/2020-Brea stfeeding-Report-Card-H.pdf.

84. Bagci Bosi AT, Eriksen KG, Sobko T, et al. Breastfeeding practices and policies in WHO European region member states. Public Health Nutr. 2016;19:75364.

85. World Health Organization. World health statistics 2013. Geneva: WHO 2013.

86. Brown $\sqcup$, Sear R. Are mothers less likely to breastfeed in harsh environments? Physical environmental quality and breastfeeding in the born in Bradford study. Matern Child Nutr. 2019;15(4):e12851.

87. Singh GK, Kogan MD, Dee DL. Nativity/immigrant status, race/ethnicity, and socioeconomic determinants of breastfeeding initiation and duration in the United States, 2003. Pediatrics. 2007;119(Suppl 1):S38-46.

88. World Health Organization and UNICEF. Global strategy for infant and young child feeding. https://www.who.int/maternal_child_adolescent/ documents/9241562218/en/. (Accessed 10 Sept 2020).

89. World Health Organization. Global initiative for childhood cancer. https:// www.who.int/cancer/childhood-cancer/en/.

90. Hanson LA. The mother-offspring dyad and the immune system. Acta Paediatr. 2000;89:252-8.
91. Mossberg AK, Hun MK, Morozova-Roche LA, et al. Structure and function of human alpha-lactalbumin made lethal to tumor cells (HAMLET)-type complexes. FEBS J. 2010;277:4614-25.

92. Hassiotou F, Hartmann PE. At the dawn of a new discovery: the potential of breast milk stem cells. Adv Nutr. 2014;5:770-8.

93. Greaves MF. Aetiology of acute leukaemia. Lancet. 1997;349:344-9.

94. Rogier EW, Frantz AL, Bruno ME, et al. Secretory antibodies in breast milk promote long-term intestinal homeostasis by regulating the gut microbiota and host gene expression. Proc Natl Acad Sci U S A. 2014;111(8):3074-9.

95. Andreas NJ, Kampmann B, Mehring L-DK. Human breast milk: a review on its composition and bioactivity. Early Hum Dev. 2015;91(11):629-35.

96. Stewart CJ, Ajami NJ, O'Brien JL, et al. Temporal development of the gut microbiome in early childhood from the TEDDY study. Nature. 2018; 562(7728):583-8.

97. DeWeerdt S. How baby's first microbes could be crucial to future health. Nature. 2018;555(7695):S18-9.

\section{Publisher's Note}

Springer Nature remains neutral with regard to jurisdictional claims in published maps and institutional affiliations.
Ready to submit your research? Choose BMC and benefit from:

- fast, convenient online submission

- thorough peer review by experienced researchers in your field

- rapid publication on acceptance

- support for research data, including large and complex data types

- gold Open Access which fosters wider collaboration and increased citations

- maximum visibility for your research: over $100 \mathrm{M}$ website views per year

At $\mathrm{BMC}$, research is always in progress.

Learn more biomedcentral.com/submissions 